\title{
Genetic Influences on Brain Gene Expression in Rats Selected for Tameness and Aggression
}

\begin{abstract}
Henrike O. Heyne, ${ }^{*,+1}$ Susann Lautenschläger, ${ }^{\dagger}$ Ronald Nelson, ${ }^{\ddagger}$ François Besnier, ${ }^{\ddagger \S \S}$ Maxime Rotival, ${ }^{* *}$ Alexander Cagan, ${ }^{*}$ Rimma Kozhemyakina, ${ }^{\dagger \dagger}$ Irina Z. Plyusnina, ${ }^{\dagger+, 2}$ Lyudmila Trut, ${ }^{\dagger \dagger}$ Örjan Carlborg, ${ }^{\ddagger}$

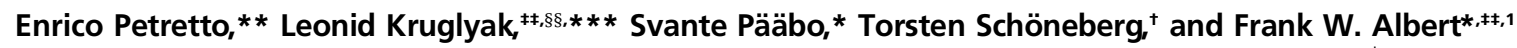
*Department of Evolutionary Genetics, Max Planck Institute for Evolutionary Anthropology, 04103 Leipzig, Germany, ${ }^{\dagger}$ Institute for Biochemistry, University of Leipzig, Medical Faculty, 04103 Leipzig, Germany, ${ }^{\ddagger}$ Swedish University of Agricultural Sciences, Department of Clinical Sciences, Division of Computational Genetics, Box 7078 SE-75007 Uppsala, Sweden, §Section of Population Genetics and Ecology, Institute of Marine Research, Havforskningsinstituttet, Postboks 1870, Nordnes 5817, Bergen, Norway, ${ }^{* *}$ MRC Clinical Sciences Centre, Faculty of Medicine, Imperial College London, London W12 ONN, United Kingdom, ${ }^{+{ }^{\dagger}}$ Institute of Cytology and Genetics, Siberian Branch of the Russian Academy of Sciences, 630090 Novosibirsk, Russia, ${ }^{\ddagger}$ Department of Human

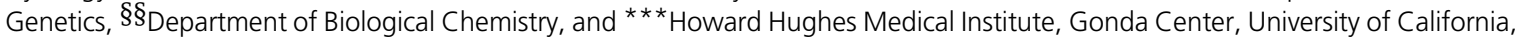

Los Angeles, California 90095
\end{abstract}

\begin{abstract}
Interindividual differences in many behaviors are partly due to genetic differences, but the identification of the genes and variants that influence behavior remains challenging. Here, we studied an $F_{2}$ intercross of two outbred lines of rats selected for tame and aggressive behavior toward humans for $>64$ generations. By using a mapping approach that is able to identify genetic loci segregating within the lines, we identified four times more loci influencing tameness and aggression than by an approach that assumes fixation of causative alleles, suggesting that many causative loci were not driven to fixation by the selection. We used RNA sequencing in $150 \mathrm{~F}_{2}$ animals to identify hundreds of loci that influence brain gene expression. Several of these loci colocalize with tameness loci and may reflect the same genetic variants. Through analyses of correlations between allele effects on behavior and gene expression, differential expression between the tame and aggressive rat selection lines, and correlations between gene expression and tameness in $\mathrm{F}_{2}$ animals, we identify the genes $\mathrm{Gltscr2}$, Lgi4, Zfp40, and S/c17a7 as candidate contributors to the strikingly different behavior of the tame and aggressive animals.
\end{abstract}

EHAVIORAL differences among members of a species are in part due to genetic variation. The identification of the genes and variants that influence behavior remains challenging. In human genome-wide association studies of psychiatric and cognitive traits, the identified loci typically explain only a small fraction of the heritability, i.e., the additive genetic contribution

Copyright $\odot 2014$ by the Genetics Society of America

doi: $10.1534 /$ genetics.114.168948

Manuscript received July 29, 2014; accepted for publication August 22, 2014; published Early Online September 3, 2014.

Supporting information is available online at http://www.genetics.org/lookup/suppl/ doi:10.1534/genetics.114.168948/-/DC1.

Raw sequencing reads as well as expression data, inferred genotypes, and genotype probabilities are available in the ArrayExpress archive (http://www.ebi.ac.uk/ arrayexpress/) as accession no. ERP006055. We will provide IBD matrices upon request.

${ }^{1}$ Corresponding authors: Institute for Biochemistry, University of Leipzig, Medical Faculty, Johannisallee 30, 04103 Leipzig, Germany. E-mail: henrikeheyne@hotmail.de; and Department of Human Genetics, Gonda Center, 695 Charles E. Young Dr. S., University of California, Los Angeles, CA 90095. E-mail: falbert@mednet.ucla.edu 2..Z.P. deceased. to the trait (Watanabe et al. 2007; Hovatta and Barlow 2008; Deary et al. 2009; Otowa et al. 2009; Calboli et al. 2010; Terracciano et al. 2010; Flint and Munafo 2013; Rietveld et al. 2013; Sokolowska and Hovatta 2013). In experimental crosses in model species, especially between inbred lines, the identified loci (termed quantitative trait loci, QTL), often explain much more of the heritability (Lynch and Walsh 1998). However, in this design the spatial resolution is limited-the QTL are wide and contain many, sometimes hundreds of genes. With the exception of a handful of identified genes with quantitative effects on behavioral traits (Yalcin et al. 2004; Watanabe et al. 2007; McGrath et al. 2009; Bendesky et al. 2012; Goodson et al. 2012), gene identification is particularly difficult for behavioral QTL that often have modest effect sizes (Flint 2003; Willis-Owen and Flint 2006; Wright et al. 2006; Hovatta and Barlow 2008).

The causal variants within a QTL can alter the protein sequence encoded by a gene or affect gene expression. Such 
regulatory variants can be identified as "expression QTL" (eQTL). An attractive approach for nominating candidate genes underlying QTL is therefore to identify genes within the QTL regions whose expression is influenced by an eQTL in a tissue of relevance to the phenotype (Hubner et al. 2005; Petretto et al. 2006b; Mackay et al. 2009). This approach has been successfully applied to quantitative traits in different species, e.g., yeast (Perlstein et al. 2007), rats (Hubner et al. 2005; Heinig et al. 2010), mice (Mehrabian et al. 2005; Schadt et al. 2005), and humans (Moffatt et al. 2007; Musunuru et al. 2010). A few eQTL studies have also recently been carried out to identify candidate genes for behavioral QTL (Hitzemann et al. 2004; De Jong et al. 2011; Saba et al. 2011; Kelly et al. 2012).

Here, we studied two lines of rats (Rattus norvegicus) that, starting from one wild population, have been selected for increased tameness and increased aggression toward humans, respectively. These experimental populations derive from the work of Dmitry K. Belyaev who, along with his famous related work in foxes (Belyaev 1979; Trut 1999), initiated the rat lines to study the evolution and underlying biological mechanisms of tameness as a crucial first step in animal domestication (Diamond 1999; Trut 1999; Wiener and Wilkinson 2011). For $>64$ generations, the tame rats were selected exclusively based on whether they tolerate or even seek out human contact, while the aggressive rats were selected to vigorously defend themselves from any attempted handling. This strong selection has resulted in two lines that respond extremely differently to attempts of handling. Whereas the aggressive rats attack and try to escape, the tame rats lack fear or aggression. They tolerate handling and sometimes actively explore the approaching hand. The strong behavioral selection has resulted in a number of differences in morphology, neurotransmitters, and hormone levels (Naumenko et al. 1989; Plyusnina and Oskina 1997; Albert et al. 2008).

Cross fostering experiments excluded postnatal maternal effects as a major influence on tameness and aggression in these animals, suggesting a substantial genetic contribution to the behavior (Albert et al. 2008; Plyusnina et al. 2009). To gain insight into the genetic basis of tameness and associated phenotypes, we earlier bred an $\mathrm{F}_{2}$ intercross between the tame and aggressive rat lines (Albert et al. 2009). Genetic mapping in 700 rats from this population revealed two genome-wide significant QTL for tameness. Three additional loci were found as part of an epistatic network influencing tameness (Albert et al. 2009). However, these QTL harbor hundreds of genes, and individual genes influencing the behavior remain unknown.

The tame and the aggressive selection lines originate from one wild population. Throughout selection, the populations were kept as outbred as possible by avoiding matings between close relatives. Together with the limited number of generations and the modest population sizes since initiation of the lines, these characteristics make it unlikely that tameness and aggression are mainly caused by new mutations arising after the animals were caught. Instead, most genetic variants that were selected are expected to be due to standing variation already present in the wild population. We earlier confirmed the existence of considerable DNA sequence variation both between and within each of the two lines (Albert et al. 2011). Therefore, it seems plausible that alleles influencing behavior segregate not only between but also within the lines. QTL analyses that assume fixation of causative alleles could miss important sources of genetic variation.

Here, we used mRNA sequencing to quantify gene expression in the brains of 150 rats from the $\mathrm{F}_{2}$ intercross population. These data were used to map eQTL influencing differences in expression levels, using a QTL mapping method (flexible intercross analysis, FIA) (Rönnegård et al. 2008) that can account for segregating variation within the founders of a cross between two outbred lines. Using FIA, we also found four times as many loci influencing tameness than previously seen (Albert et al. 2009). We identify multiple genes within the tameness QTL whose expression in the brain is influenced by the genetic variants from the tame- and aggressive lines. By combining correlations of allele effects on behavior and gene expression, differential brain gene expression analyses between the tame and aggressive rat lines and correlation with tameness in $\mathrm{F}_{2}$ animals, we prioritize genes whose altered expression may be influencing tameness.

\section{Materials and Methods}

\section{Rat populations}

The tame and aggressive rat populations are derived from a long-term selective breeding experiment that was initiated in 1972 in the Institute of Cytology and Genetics in Novosibirsk, Russia (Naumenko et al. 1989). Two rat ( $R$. norvegicus) lines were selected from one wild-caught population for the single criterion of increased tameness and increased aggression when confronted with a human hand. The original population consisted of $\sim 200$ animals. At each generation, the 30\% most tame and most aggressive individuals were selected for further breeding. Inbreeding was avoided in both strains by avoiding mating between close relatives. In 2005, subpopulations from both lines were transferred to Leipzig to study the genetic basis of the tameness (Albert et al. 2008, 2009, 2011, 2012). During all care-taking procedures and experiments, animals from different lines and generations were treated identically. The study was approved by the regional government of Saxony (TVV Nr. 29/95).

\section{Selection of $F_{2}$ animals based on extreme tameness/ aggression}

To perform genetic mapping of loci influencing tameness and aggression, we have earlier generated an $\mathrm{F}_{2}$ intercross population of $\sim 700$ rats from a cross between the tame and the aggressive rats (Albert et al. 2009). Briefly, in that study, $\mathrm{F}_{2}$ rats were assigned a "tameness score" based on a behavioral test (Albert et al. 2008) where rats were exposed to a standardized handling procedure, and their responses were videotaped and scored. To summarize a rat's behavior in this test, principal component analysis (PCA) was performed on a set of 11 individual 
measures. The first principal component provides a quantitative measurement for tameness (Albert et al. 2009). For the present study, we used stored organs from the 75 tamest and the 75 most aggressive $\mathrm{F}_{2}$ animals.

\section{Tissue collection}

Animals were earlier anesthetized by $\mathrm{CO}_{2}$ and killed by cervical dislocation (Albert et al. 2009). Tissues were collected, snap frozen in liquid nitrogen, and stored at $-80^{\circ}$. Before freezing, each brain was dissected to separate the brainstem and cerebellum from the fore- and midbrain.

For the current study, brains were cut with a scalpel along the midsagittal plane in two hemispheres at $-20^{\circ}$. Before dissection, tissues were left to equilibrate to $-20^{\circ}$ for at least half an hour to obtain the best consistency during cutting. To protect RNA quality, brains were never completely thawed during dissection. Complete right brain hemispheres comprising tel-, di- and mesencephalon were used. Frozen hemispheres were homogenized in $5 \mathrm{ml}$ of TRIzol (Invitrogen, Darmstadt, Germany) in glass tubes using a Schütt homogenizer with $3000 \mathrm{rpm}$ for 13 rounds. A total of $2.5 \mathrm{ml}$ of TRIzol was added afterward to obtain a final volume of $7.5 \mathrm{ml}$ of TRIzol per brain hemisphere.

\section{CDNA library preparation}

RNA was extracted by chloroform extraction and purified with the Qiagen RNEasy MinElute Clean-up kit according to the manufacturer's instructions. All RNA samples were of high quality as judged by Agilent Bioanalyzer (Agilent Technologies, Böblingen, Germany). RNA integrity numbers (RINs) (Schroeder et al. 2006) for all samples ranged from 8.5 to 10, where 10 corresponds to maximum RNA quality (Supporting Information, Table S1). RNA-seq libraries were generated according to in-house protocols; for details see Kuhlwilm et al. (2013). mRNA was extracted from $10 \mu \mathrm{g}$ of total RNA by capture on poly-T-covered magnetic beads, chemically fragmented, and used as template for cDNA synthesis using random hexamer primers. Double-stranded cDNA was blunt ended and Illumina sequencing adapters were ligated to the cDNA. To minimize potential batch effects, balanced numbers from the tame and the aggressive groups of $\mathrm{F}_{2}$ animals were processed together throughout RNA extraction and library generation and distributed evenly across sequencing runs.

\section{Sequencing}

Libraries were adjusted to a concentration of $10 \mathrm{nM}$ and individuals distributed into three different pools, each consisting of equal numbers of tame and aggressive individuals. They were then sequenced to a median coverage of 10.8 million reads per animal [standard deviation (SD) \pm 0.8 million reads per animal] on Illumina Genome Analyzer 2 machines (Illumina, San Diego) with 76-bp single end reads. We called bases using Ibis (Kircher et al. 2009). Adapter sequences were trimmed, adapter dimers excluded, and the reads filtered for quality. Raw sequencing reads are available in the ArrayExpress archive (http:// www.ebi.ac.uk/arrayexpress/) as accession no. ERP006055.

\section{Read mapping and gene expression quantification}

Reads were mapped to the rat genome (assembly and gene annotation RGSC 3.4) with the program TopHat, version 1.4.1 (Trapnell et al. 2009). We ran TopHat with the transcriptome index option and provided the program with a transcriptome sequence file obtained from Ensembl release 69. With this option, TopHat first aligns reads to the virtual transcriptome before mapping unmapped reads to the whole genome.

Gene expression was then quantified using the program Cufflinks (Trapnell et al. 2010) version 1.3.0, with the following parameters: We employed the fragment bias correction algorithm, which aims to account for sequence-specific biases introduced by the use of random hexamer primers (Roberts et al. 2011). We further provided Cufflinks with the median read length for each library as measured with the Agilent BioAnalyzer before sequencing. Further, we supplied Cufflinks with a reference annotation file to quantify whole gene and different isoform expression, using the Ensembl release 69 gene annotation. Any alignments not compatible with any reference transcripts were ignored. We thus did not search for novel genes and did not attempt to alter the gene models represented in the Ensembl annotation. Unless otherwise noted, we used fragments per kilobase of transcript per million fragments mapped (FPKM) expression values (Trapnell et al. 2010) for further analyses. In FPKM, the RNA-seq data are normalized to the length of each gene and the total number of aligned reads in the library (Trapnell et al. 2010). FPKM values were transformed using log2 (FPKM + 1). Expression data are available in the ArrayExpress archive (http://www.ebi.ac.uk/arrayexpress/) as accession no. ERP006055. All further analyses were done in the R environment (R Development Core Team 2012).

\section{Gene expression preprocessing}

We retained for analyses all genes for which Cufflinks reported expression levels, resulting in quantifications for 24,139 genes. To avoid confounding by potential batch effects (Leek et al. 2010), we corrected the expression data for batches defined by groups of samples processed on the same date (Table S1). This was done by fitting a linear model with gene expression as the response variable and batch as the predictor variable. We used the residuals of the model as batch-corrected gene expression traits. To additionally account for unknown unwanted sources of variation, we computed surrogate variables of the gene expression matrix by using the R package Surrogate Variable Analysis (SVA) (Leek and Storey 2007; Leek et al. 2012). We used the two surrogate variables reported by the software as covariates for expression QTL mapping. We used the phenotypes tameness and adrenal gland weight (Albert et al. 2009) as covariates when calculating the surrogate variables. To rule out the possibility that we might accidentally correct for true genetic regulators that might influence many gene expression traits, we also performed QTL mapping for the values of the two surrogate variables. The two SVA did not significantly map to any region of the genome. SVA-corrected expression data yielded more eQTL than either a correction 
for sex or a correction for sex and RNA library processing batch (Table S2). We also used the animals' sex as a covariate during eQTL mapping.

\section{Genetic map construction}

Genotypes at 152 microsatellite and 49 single-nucleotide polymorphism (SNP) markers were used to build a 1728-cM genetic map as in Albert et al. (2009). Genotypes, genetic markers, marker positions, and pedigree data were the same as for the QTL mapping of tameness described previously in Albert et al. (2009). The probability of each $\mathrm{F}_{2}$ allele to originate from either the tame or the aggressive line were recomputed using an updated algorithm where haplotypes and missing marker information were inferred iteratively to reconstruct any missing genotypes. First, haplotypes were inferred from genotype and pedigree data with a custom implementation of the method described in Besnier and Carlborg (2009). In our implementation, haplotype reconstruction steps were alternating with missing genotype inference steps. The two steps were run iteratively for each pedigree generation, in turn, until no new haplotype or missing genotype could be inferred. The probability of each $\mathrm{F}_{2}$ allele to originate from either the tame or the aggressive line were then reinferred from phased genotype and pedigree, using the identity by descent (IBD) matrix calculated as in Pong-Wong et al. (2001) and implemented in Besnier and Carlborg (2007). We will provide IBD matrices upon request.

\section{eQTL mapping using Haley Knott regression}

We searched for eQTL on autosomes in steps of $1 \mathrm{cM}$ using Haley Knott regression (HKR) (Haley and Knott 1992) as implemented in the $\mathrm{R}$ package $\mathrm{R} / \mathrm{qtl}$ (Broman et al. 2003). The outbred cross dataset was prepared for use in R/qtl using the $\mathrm{R}$ package qtl.outbred (Nelson et al. 2011). Inferred genotypes and genotype probabilities are available in the ArrayExpress archive (http://www.ebi.ac.uk/arrayexpress/) as accession no. ERP006055. We used logarithm of odds (LOD) scores to quantify how strongly genetic variation across the genome influences the expression of a gene. To determine the significance threshold for QTL, we performed 1000 permutations across all 14,000 genes with sufficiently abundant expression (see below) by randomizing genotypes with respect to phenotypes and performing full genome scans for the permuted datasets. To calculate false discovery rates (FDRs), we divided the number of expected eQTL (as determined from the permutations) by the number of eQTL observed in the real data at a given LOD threshold (Benjamini and Yekutieli 2005). We found that a LOD threshold of 5.28 results in an FDR of 5\% and used this value as the significance threshold for further analyses. We report confidence intervals (CIs) for QTL location as 1.8-LOD drop intervals as recommended for $\mathrm{F}_{2}$ intercross designs in Broman and Saunak (2009).

\section{Influence of sequencing depth on eQTL mapping}

To evaluate to what extent sequencing depth influences the number of detected eQTL, we randomly selected 5000 genes, downsampled their expression data to different sequencing depths, and repeated the eQTL mapping in these trimmed datasets. At each depth, we randomly permuted genotypes relative to phenotypes 10 times across the 5000 genes and determined the $99.9 \%$ quantile of the resulting distribution of LOD scores. This LOD score was used as the significance threshold for eQTL detection at the given depth. We calculated false discovery rates and recorded the number of eQTL that would have been found had we sequenced to this depth of coverage using the same procedure as described above (Figure S1).

\section{False discovery rates as a function of gene expression level}

To assess at which expression level genes can be measured accurately enough to give robust eQTL mapping results we calculated false discovery rates in bins of increasing gene expression levels (Figure S2). Genes were grouped according to their expression level into 24 bins, each containing 1000 genes, except for the bin with the lowest expression, which contained 1139 genes. We performed 10 permutations within each of the 24 bins, determined the $99.9 \%$ quantile of permuted LOD scores, and used this LOD score as the eQTL detection threshold for the given bin. We found that the FDR stayed consistently $<5 \%$ among the 14,000 most highly expressed genes (Figure S2). At a 5\% FDR, we identified 3.2 eQTL per 100 genes when considering the 14,000 highest expressed genes as opposed to 1.7 eQTL per 100 genes when considering all genes. For all later analyses, we only used the 14,000 most highly expressed genes.

\section{Flexible intercross analysis}

Flexible intercross analysis (FIA) (Rönnegård et al. 2008) is a QTL analysis method for analyses of data from intercross populations that does not assume that the founder lines are fixed for alternative QTL alleles. Here, we used FIA, as implemented in $\mathrm{R}$ scripts kindly provided by L. Rönnegård, to perform genome scans for tameness and gene expression. For tameness, significance thresholds were determined by 1000 random permutations. For expression data, hundreds of permutations for all genes were not computationally feasible even on a computer with hundreds of nodes. Instead, we approximated the score distribution for QTL under the null hypothesis using information from 30 permutations for each of 2000 randomly sampled genes. False discovery rates were estimated as described above for HKR.

We also conducted a targeted search for local eQTL by considering only the marker located closest to a given gene. This procedure increases statistical power by reducing the multiple testing burden. False discovery rates were estimated as described above, but using only permutations at the local markers.

\section{FIA QTL confidence intervals}

We defined FIA eQTL confidence intervals by using information from eQTL that were detected by both FIA and HKR. We used the "approx.fun" function in $\mathrm{R}$ to fit a function relating 


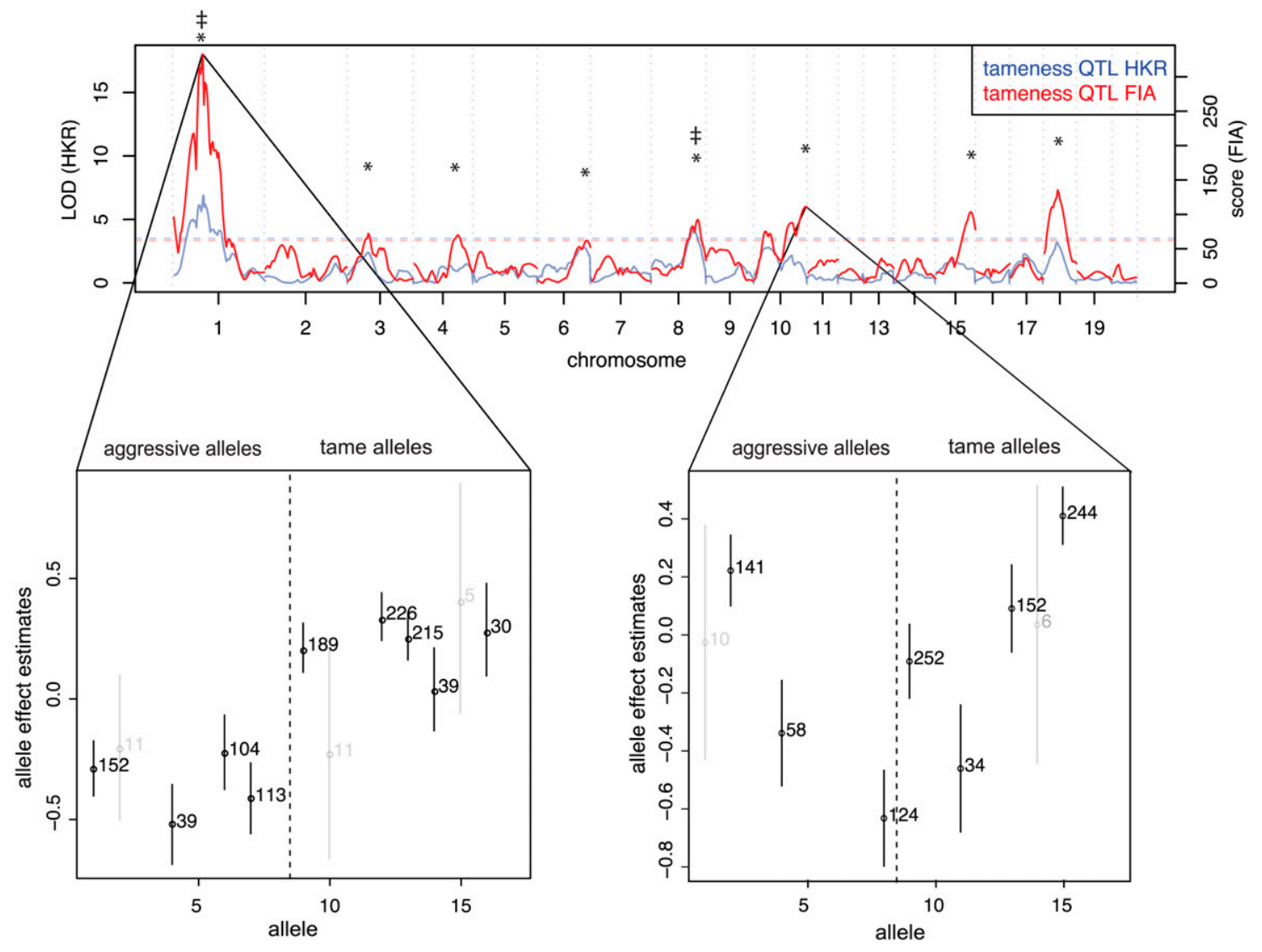

Figure 1 Tameness QTL mapping. Genome-wide statistical support for QTL affecting tameness in analyses assuming fixation of causal alleles within the founder populations (HKR, blue line) as well as analyses allowing segregation of causal alleles among founder animals (FIA, red line). Horizontal dashed lines indicate a $2 \%$ FDR significance threshold; QTL exceeding the threshold are indicated by * (FIA) and * (HKR). Allele effect plots of the FIA tameness QTL on chromosomes 1 and 10 are shown below. Circles indicate the point estimate and lines the standard errors of the allellic effects. The numbers of individuals that carry a respective allele are printed to the right of the point estimates. For clarity, the results for alleles with $<20$ observations are shown in light gray.

the FIA mapping score to the width of the HKR confidence interval. Using this function, we interpolated confidence intervals for all FIA QTL. To avoid unrealistically small confidence intervals for the most highly significant eQTL, we set the minimum size of the confidence intervals to $10 \mathrm{cM}$ (Figure S3, A-C).

We compared our FIA eQTL confidence intervals to those obtained by bootstrapping (Manichaikul et al. 2006). For each of 29 randomly selected eQTL, we computed 200 bootstraps by mapping in a dataset constructed by sampling with replacement from the 150 rats. In about half the cases, the bootstrap 95\% confidence intervals delineate a narrow peak around the eQTL peak. Most of these bootstrap confidence intervals are completely contained in the respective confidence intervals derived as described above (Figure S4). About a third of the bootstrap confidence intervals were very large, in some cases extending across the whole chromosome (Figure S4). It is known that large confidence intervals can occur when QTL confidence intervals are estimated by bootstraps, especially when genetic markers are sparse, prompting recommendations not to use bootstrapping to estimate QTL confidence intervals (Manichaikul et al. 2006). Combined with the fact that the narrow bootstrap confidence intervals agree well with our HKR-estimated CIs, this suggests that our approach described above provides a reasonable approximation for FIA eQTL confidence intervals.

eQTL were treated as local when the gene's physical position overlapped their confidence interval. Genetic positions in centimorgans were interpolated to the physical megabase scale and vice versa with the approx.fun function using coordinates given in table S2 of Albert et al. (2009) that contains the genetic and physical position of the markers that were used to build the genetic map.

eQTL and tameness QTL were treated as overlapping when their confidence intervals overlapped by at least $1 \mathrm{cM}$. In the test of whether windows within tameness QTL had more eQTL than the rest of the genome, we used nonoverlapping sliding windows with a width of $18 \mathrm{cM}$, which is the median confidence interval size of HKR eQTL.

\section{Regional clustering of eQTL}

We counted the number of genes with local eQTL in a sliding window analysis. Windows were advanced in 2-cM steps and had a width of $18 \mathrm{cM}$, which was the median confidence interval for eQTL in the HKR analysis. When calculating 
Table 1 Locations of FIA tameness QTL

\begin{tabular}{|c|c|c|c|c|c|c|}
\hline Chromosome & QTL start (cM) & QTL peak (cM) & QTL end (cM) & QTL start (Mb) & QTL peak (Mb) & QTL end (Mb) \\
\hline 1 & 40.4 & 52.0 & 63.6 & 43.7 & 69.4 & 94.1 \\
\hline 3 & 18.8 & 36.0 & 53.2 & 25.1 & 41.0 & 66.9 \\
\hline 4 & 60.8 & 78.0 & 95.2 & 107.1 & 140.0 & 163.7 \\
\hline 6 & 70.8 & 88.0 & 96.0 & 112.0 & 131.3 & 144.0 \\
\hline 8 & 65.7 & 82.0 & 98.0 & 92.6 & 106.6 & 126.8 \\
\hline 10 & 2.9 & 20.0 & 37.1 & 7.6 & 23.3 & 38.4 \\
\hline 10 & 76.4 & 92.0 & 96.0 & 90.2 & 103.3 & 106.0 \\
\hline 15 & 46.1 & 62.0 & 72.0 & 72.5 & 91.3 & 104.0 \\
\hline 18 & 9.1 & 24.0 & 38.9 & 14.6 & 48.2 & 62.7 \\
\hline
\end{tabular}

The locations of all FIA tameness QTL identified at a 2\% FDR are shown.

correlations between the numbers of genes located in an interval with the number of local eQTL, we used nonoverlapping sliding windows of $18 \mathrm{cM}$ to minimize dependencies between adjacent data points.

To test whether there are positions with more distant eQTL than expected, we determined the number of distant eQTL that would be expected to map to a given position in the genome if eQTL were positioned at random. We generated 1000 sets of random genomic positions for the distant eQTL, while keeping the number of eQTL per chromosome unchanged (Figure S5). We used the 99\% quantile of the resulting distribution as the threshold to call significant eQTL clusters.

\section{Coexpression networks}

Weighted gene coexpression network analysis (WGCNA) (Langfelder and Horvath 2008) was used on the set of 14,000 expressed genes to detect clusters. Biweight correlation was used to identify clusters robust to outliers and all other parameters were left to default. GO enrichment was performed using DAVID (Huang et al. 2008). Transcription factor binding site enrichment was performed using PASTAA (Roider et al. 2009): Sequences of $2 \times 500 \mathrm{bp}$ regions around the transcription start site of each gene were extracted from the BN genome and scored for JASPAR and transfac public transcription factor matrices. Clusters were then tested for enrichment of high binding affinity values.

\section{Correlating allele effects of tameness QTL and expression QTL}

If QTL for two different traits that are located at the same genomic position are caused by the same underlying variant, the effects of their alleles on the two traits should be correlated. To test this, we estimated the effect of each allele on a given trait as the mean phenotype of those $F_{2}$ animals that have inherited the given allele. The precision of this allele effect estimate is bounded by the structure of our experimental pedigree. Our $\mathrm{F}_{2}$ animals derive from four animals from the tame and the aggressive selection line, respectively, such that there is a maximum of 16 different alleles segregating at each position in the $\mathrm{F}_{2}$ population. Due to random segregation and variable offspring number, the founder alleles differ in the number of $\mathrm{F}_{2}$ individuals that carry them (Figure 1). The estimated effects of alleles that are inherited by many individuals are more precise than those carried by fewer individuals.
Further, some of the 16 founder alleles might be identical, so that the effective number of alleles will usually be lower than 16. We did not attempt to correct for differences in precision and did not attempt to formally group the founder alleles into functionally equivalent classes.

We performed two scans for allele effect correlations. In both scans, both local and distant eQTL were used. In the first scan, we tested for correlation between those eQTL that overlap one of the eight FIA tameness QTL. The correlations were determined at the peak positions of the tameness QTL, irrespective of the location of the respective eQTL peaks. In the second scan, we tested all eQTL, irrespective of whether they overlapped a tameness QTL. These correlations were determined at the peaks positions of the eQTL. The two scans thus differ in several respects: the set of eQTL in the analyses, the precise positions tested (these can differ between the two scans even for pairs of tameness and eQTL represented in both scans) and the corrected significance threshold (due to different numbers of tests).

\section{Differential gene expression in brains of animals from the tame and aggressive founder lines}

Right brain hemispheres were harvested from five adult male rats from the tame and from the aggressive selection lines, respectively. mRNA was extracted as described above and fluorescently labeled and hybridized to Affymetrix microarrays (GeneChip Rat Genome 230 2.0) following the manufacturer's instructions. We mapped array probes to genes using reannotated chip definition files (Dai et al. 2005). Array normalization was performed using robust multiarray average (RMA) (Irizarry et al. 2003) and genes called as expressed using the Affymetrix MAS5 algorithm. Both RMA and MAS5 were used as implemented in the R bioconductor 'affy' package (Bolstad et al. 2003). Only genes where at least four of the five animals in a group showed expression according to MAS5 were analyzed. We tested for differential expression between the tame and aggressive animals using $t$-tests. The FDR was determined by calculating all permutations that are possible in two groups of five samples. For a range of $P$-value cutoffs, we calculated the expected number of false positive tests as the median number of tests exceeding a given cutoff across permutations. The FDR was calculated as this expected number of false positive tests divided by the number of positive tests observed in the real data. 


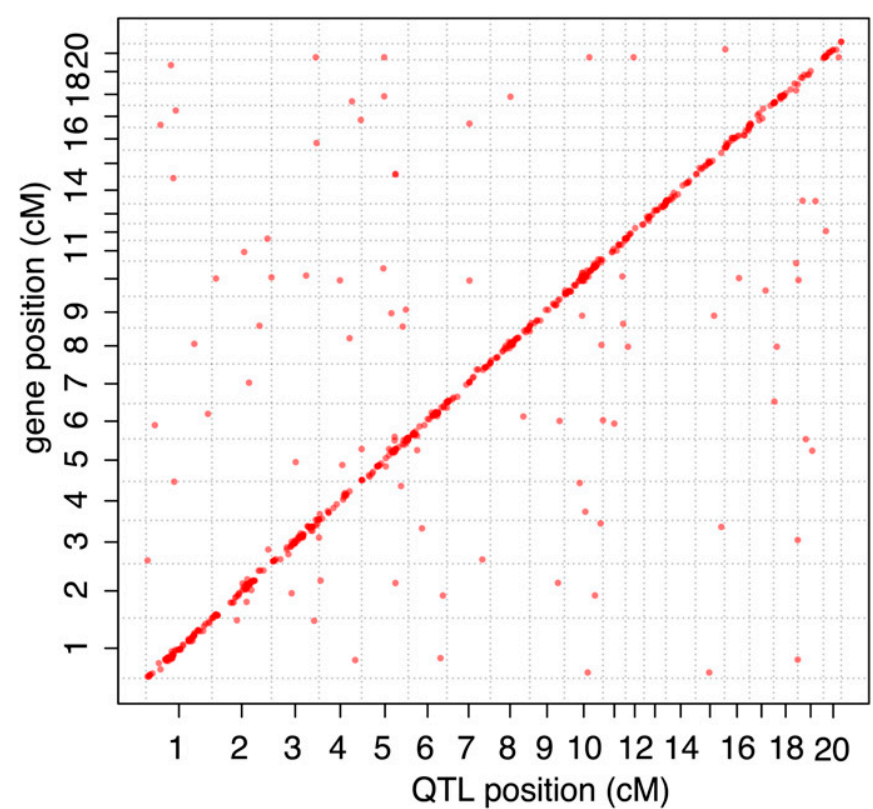

Figure 2 Brain expression QTL mapping. Locations of eQTL affecting brain gene expression in an $F_{2}$ intercross between two rat lines selected for tame and aggressive behavior toward humans. Positions of genes are plotted against the positions of eQTL identified by FIA.

\section{Correlation of gene expression levels and tameness in $F_{2}$ animals}

We tested for correlation (Spearman rank correlation) between gene expression levels and tameness. The FDR was determined by comparing the observed data to 100 permuted datasets.

\section{Results}

\section{RNA sequencing}

We selected the 75 tamest and the 75 most aggressive animals of the $700 \mathrm{~F}_{2}$ intercross rats bred for our earlier study (Albert et al. 2009) for RNA sequencing. Nonstranded Illumina sequencing libraries were generated from polyAenriched RNA from right cerebral hemispheres that were frozen as part of our earlier characterization of these animals. The RNA-seq libraries were sequenced to a median coverage of 10.8 million reads per animal (standard deviation, $\mathrm{SD}=0.8$ million reads). Of these, an average of 9.0 million reads ( $83 \%$ of total reads) mapped to the rat genome (SD $=0.6$ million reads). Out of these, an average of $61.7 \%(\mathrm{SD}=2.1 \%)$ mapped unambiguously to known exons. Down-sampling analyses were used to evaluate the power of the study, and although these suggest that more eQTL could be found by increasing sequencing coverage (Figure S1), the available coverage is expected to be sufficient to discover hundreds of eQTL. Our strategy of selecting $\mathrm{F}_{2}$ animals with extreme behaviors did not lead to notably skewed or bimodal gene expression distributions (Figure S6), suggesting that this strategy does not complicate eQTL mapping.

\section{Fixed genetic differences between the tame and aggressive rat populations influence brain gene expression levels}

We began our search for eQTL using a method that assumes that the two founder populations are fixed for QTL alleles with different effects on expression (HKR) (Haley and Knott 1992); this method was also used in our earlier mapping study (Albert et al. 2009). Because the accuracy of RNA-seq increases with increasing gene expression abundance, we limited our search to genes where eQTL can be detected with high statistical power (see Materials and Methods). In total, 443 eQTL were detected at a FDR of 5\% using HKR (Table S3). Thus, genetic differences between the tame and the aggressive rats result in mRNA expression differences at hundreds of genes.

\section{Genetic variation within the selection lines influences tameness and brain gene expression levels}

To explore the contribution of within-line genetic variation on behavior and gene expression, we used FIA (Rönnegård et al. 2008), a QTL mapping method that is able to detect loci that are not fixed between the tame and aggressive founders.

We first applied FIA to tameness and aggression in all $700 \mathrm{~F}_{2}$ animals and found eight QTL affecting tameness and aggression at a $2 \%$ permutation-based FDR (Figure 1, Table 1). These QTL include the two previously identified QTL (Albert et al. 2009). At the most significant FIA tameness QTL on chromosome 1 (corresponding to the "Tame-1" locus identified in Albert et al. (2009), the alleles carried by the tame and the aggressive individuals used to found the $F_{2}$ pedigree have clearly different effects on tameness (Figure 1). However, more than one "aggressive" allele appears to exist among the four aggressive founder animals (Albert et al. 2011), and one of the four tame founders appears to be heterozygous for one "tame" and one aggressive allele. Nevertheless, the overall allelic differentiation between the tame and the aggressive founder individuals explains why this locus was also the strongest QTL identified by HKR. By contrast, the FIA QTL at the end of chromosome 10 that had the third highest significance did not correspond to a HKR QTL (Figure 1). The allelic effects at this locus are highly heterogeneous, with at least one tame and one aggressive allele segregating in both the tame and the aggressive founder animals. Thus, allowing causative alleles to segregate within the lines quadruples the number of detected tameness QTL, suggesting that the genetic basis of this behavior is considerably more complex than previously appreciated.

Next, we asked how segregating allelic variation affects variation in brain gene expression levels. When using FIA for eQTL mapping, we detected 689 FIA eQTL influencing the expression of 670 genes at an FDR of 5\% (Figure 2, Figure S7, Table S4). Of these, 411 were also detected by HKR. Conversely, of the $443 \mathrm{HKR}$ eQTL, all but 32 were detected by FIA. These 32 eQTL had low LOD scores close to the genomewide significance threshold, lower than the HKR eQTL identified with both HKR and FIA (Figure S8A, Wilcoxon rank 


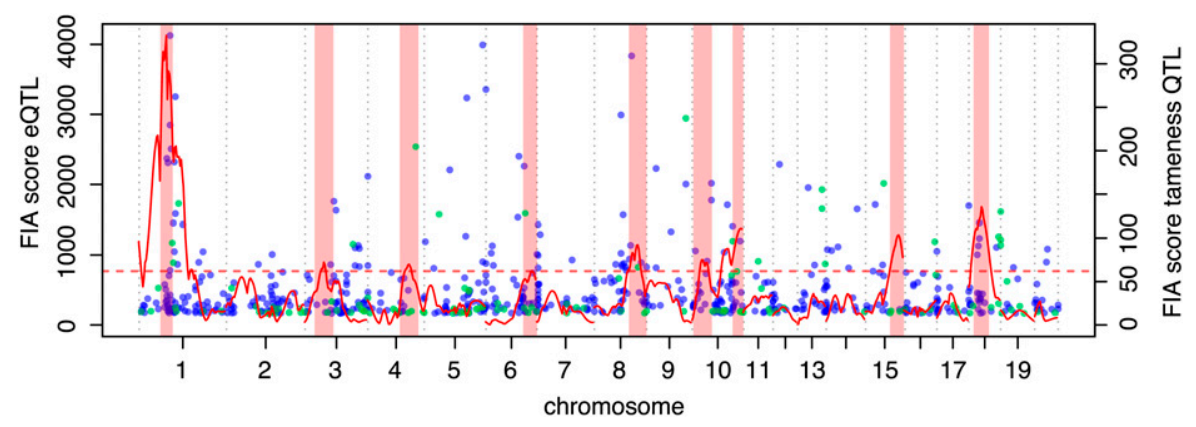

Figure 3 Genome-wide overlap of QTL affecting tameness and eQTL affecting gene expression in the brain. Red solid line, FIA scores for tameness QTL mapping; light red rectangles, confidence intervals for tameness QTL locations. FIA eQTL at a 5\% FDR are shown as dots. Blue, local eQTL; green, distant eQTL; red dashed horizontal line, 2\% FDR score threshold for tameness QTL.

test: $P=6.9 \mathrm{e}-14$ ). To be conservative, we excluded the 32 eQTL identified only by HKR from further analyses. While eQTL that were found only by FIA had, on average, lower scores than eQTL found with both HKR and FIA (Wilcoxon rank test $P<2.2 \mathrm{e}-16$ ), many of them had FIA mapping scores that were highly significant (Figure S8B). Thus, FIA increased the number of detected eQTL by $68 \%$, including some loci with strong effects on gene expression that were missed by HKR because their alleles segregate within the selection lines.

\section{Genomic architecture of mRNA expression}

Of the 689 FIA eQTL (Table S4), 561 were local eQTL close to the genes they influenced and 128 were distant eQTL that did not overlap the position of the affected gene. Local eQTL had higher mapping scores than distant loci (Wilcoxon rank test, $P=1.7 \mathrm{e}-07$ ). This is consistent with previous work showing that while both local and distant eQTL exist, local eQTL typically have stronger effects (Petretto et al. 2006a; Mackay et al. 2009; Van Nas et al. 2010). A targeted search for local eQTL where we tested only the marker situated closest to a given gene revealed local eQTL at $12 \%$ of the genes tested, for a total of 1161 local eQTL (Table S5).

Clusters of trans-eQTL can be indicative of networks of coregulated genes under common genetic control by a single locus (Hubner et al. 2005; Heinig et al. 2010; Langley et al. 2013). We therefore explored whether the distant eQTL are enriched in certain regions of the genome. One region contained more distant eQTL than expected if the eQTL were distributed randomly throughout the genome. This excess was, however, small (seven eQTL per window, six or fewer expected by chance, Figure S4A). Further, the region did not overlap with any tameness QTL (Figure S4B). To directly investigate coexpression networks and their genetic regulation, we applied WGCNA (Langfelder and Horvath 2008). The method grouped 12,616 of the 14,000 genes in the analyses into 54 coexpression clusters (Table S6; Supplementary Note). For each cluster, we computed the first principal components of the expression levels of the genes in the cluster and asked how they relate to tameness. The expression of five clusters was correlated with tameness across the $150 \mathrm{~F}_{2}$ animals (Table S6). Further, mapping the expression of the 54 clusters using FIA revealed QTL for several of the clusters, but none of these QTL overlapped with a tameness QTL.

\section{Overlap of QTL affecting tameness and gene expression in the brain}

Genes whose expression levels in the brain are influenced by local eQTL that overlap tameness QTL are candidates for contributing to the differences in behavior. Of the 689 FIA eQTL, 212 (influencing the expression of 207 genes) overlapped with a tameness QTL (Figure 3). The genomic region spanned by Tame-1 (the most significant tameness QTL) contained more eQTL than other regions of similar size in the rest of the genome (Wilcoxon rank test, $P=0.007$ ). This observation is a reflection of the fact that Tame-1 is located in the most gene-dense region of the linkage map (Figure S9).

Overall, the scores of those eQTL that overlapped a tameness QTL were not different from those that did not (Wilcoxon rank test, $P$-value $=0.4$; Figure 3 ). However, the Tame-1 region was enriched for highly significant eQTL (Figure 3 and Figure 4). For example, 11 of the 79 eQTL (14\%) with a score of at least 1000 were located in Tame-1, although this locus contains only $3 \%$ of the 14,000 genes in the analyses. Thus, while many eQTL overlapped a tameness QTL, the strongest tameness QTL Tame-1 stands out in that it spans the region with the highest gene density in the genome (Figure S9) and also contains many strong local eQTL.

\section{Allele effect correlations between tameness QTL and $e Q T L$}

An overlap of QTL for tameness and gene expression does not imply that the two QTL are necessarily caused by the same sequence variants. However, where there are multiple alleles at FIA QTL, a correlation between the allele effects of overlapping tameness QTL and eQTL is expected if they are driven by the same genetic variants. If the overlap is with a local eQTL, causative variation at the gene itself may underlie the tameness QTL. If the overlap is with a distant eQTL, the gene regulated in trans need not itself contain sequence variants that influence tameness, but its expression may be part of the biological pathways that shape this behavior.

We performed two scans for allele effect correlations. First, we conducted a targeted analysis of the FIA tameness QTL and the 212 FIA eQTL that overlap them. In this scan, the allele effects of 29 genes correlated significantly with the tameness allele effects [Benjamini Hochberg $(\mathrm{BH})$ adjusted $P<0.05$, Figure 5A]. Of these 29 correlations, 25 involved Tame-1, and 3 involved distant eQTL (Table S4). 


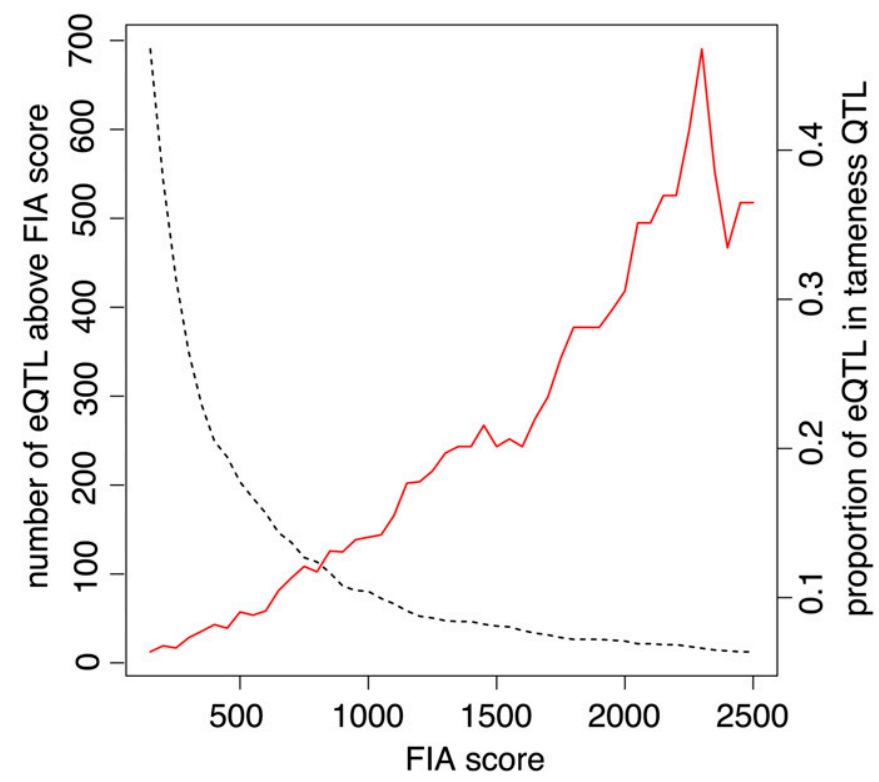

Figure 4 Enrichment of highly significant eQTL at the Tame-1 locus. The proportion of local and distant eQTL overlapping with Tame-1 (red line) at a given significance threshold ( $x$-axis) is compared to the total number of all eQTL reaching the given level of significance (dashed black line).

To explore if these allele effect correlations were specific to the significant tameness QTL, we conducted a second scan in which we tested all local eQTL for allele effect correlations with tameness, irrespective of the evidence for a tameness QTL at the respective position. Of 23 significant correlations, $18(78 \%)$ occurred at tameness QTL. Thirteen of these involved eQTL that overlapped Tame-1, and 2 additional correlations involved eQTL for genes situated very close to Tame-1 (Figure 5B). Thus, correlations between allele effects on tameness and on gene expression mostly occur at loci with significant effects on the behavior.

\section{Correlations between gene expression levels and tameness}

Across the $150 \mathrm{~F}_{2}$ animals, we found four genes whose expression levels correlated significantly with tameness (correlation strength exceeds that seen in any of 100 permutations, Figure S6, Table S7). Of these, the expression of Gltscr2 and Zfp40 correlated positively with tameness. Both genes had eQTL at the Tame-1 peak and showed allele effect correlations with Tame-1 (Figure 6). The other two genes did not have a significant eQTL.

\section{Differential expression between the tame and aggressive selection lines}

We searched for differentially expressed genes between the tame and the aggressive rat lines using two datasets: a published RNA-seq dataset of frontal cortex tissue (Albert et al. 2012) and a microarray-based comparison of cerebral hemispheres first reported here. We found 11 genes that were differentially expressed between the tame and aggressive founder animals and also had eQTL overlapping with tameness
QTL (Table S4). Five of these genes (Dhdh, Slc17a7, Lgi4, Gltscr2, and Dll3) also showed significant allele effect correlations with a tameness QTL (Figure 6, Table S8).

There was one gene (Gltscr2) that satisfied all the criteria we considered for nominating candidate genes for influencing tameness. In addition, several genes satisfied two or more criteria (Figure 6, Table S8).

\section{Discussion}

We have explored the influence of genetic variation on brain gene expression levels in a cross between two outbred lines of rats that had been selected from a common base population for strong tame and aggressive behavior toward humans. We further examined how this regulatory gene-expression variation relates to genetic influences on tameness and aggression and used the expression data to prioritize genes that may play a causal role in these behaviors.

We used two QTL mapping approaches that differ in their assumptions about the genetic homogeneity of the founder lines. The HKR assumes fixation for alternative causal alleles in the divergent founders. Using this method, we identified two tameness QTL (Albert et al. 2009) and hundreds of eQTL. Because the two selection lines are outbred and display considerable within-line genetic variation (Albert et al. 2011), we also used a QTL mapping method that does not require causative alleles to be fixed between the tame and aggressive founders (Rönnegård et al. 2008). Using this method, many additional tameness QTL and eQTL were detected. These results indicate that the genetic architecture of behavior in this cross is complex and influenced by multiple QTL, a majority of which have alleles that still are segregating in the founders. Further, there are profound differences in the brain transcriptome that are under genetic control.

Rodent behavioral QTL studies typically identify multiple QTL with small effect sizes of $<10 \%$ of the phenotypic variance of the trait (Flint 2003; Willis-Owen and Flint 2006). For example, a QTL study in heterogenous stock mice derived from eight progenitor strains identified 205 loci contributing to anxiety (Goodson et al. 2012). As a QTL needs to exceed a certain effect size to be detectable, the number of tameness QTL we have identified is an underestimate of the number of loci contributing to the phenotypic differences between the lines. Based on work on complex traits in other species, we might expect many more loci with effects too small to be detected in our current sample to influence a complex behavioral trait like tameness (e.g., Yang et al. 2010; Hunt et al. 2013). Further, selection experiments in multiple species (Drosophila (Burke et al. 2010), maize (Laurie et al. 2004), and chickens (Johansson et al. 2010; Pettersson et al. 2013) demonstrated that many loci typically respond to strong selection, as expected when trait variation is due to variants at many genes. It thus seems plausible that many genetic variants with small effects contribute to differences in several biological pathways that together shape complex behavioral traits such as tameness and defensive aggression. Epistatic 


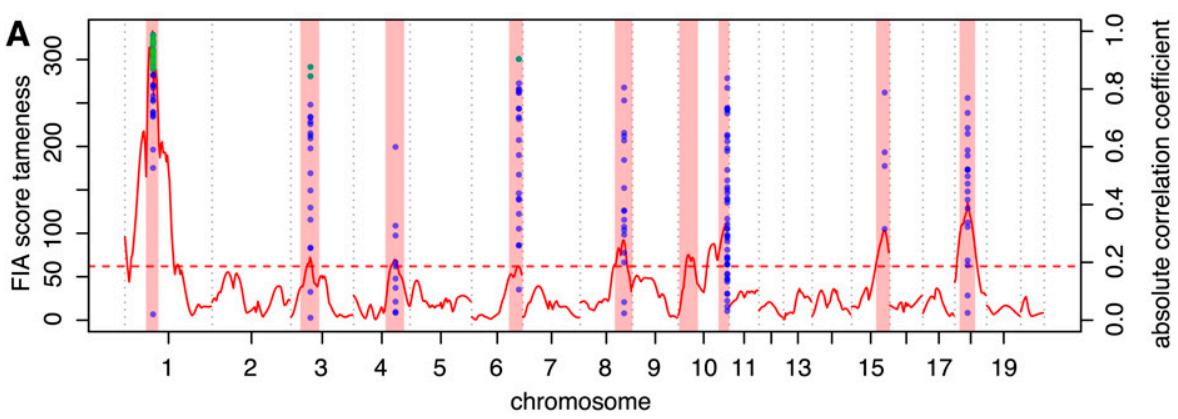

B

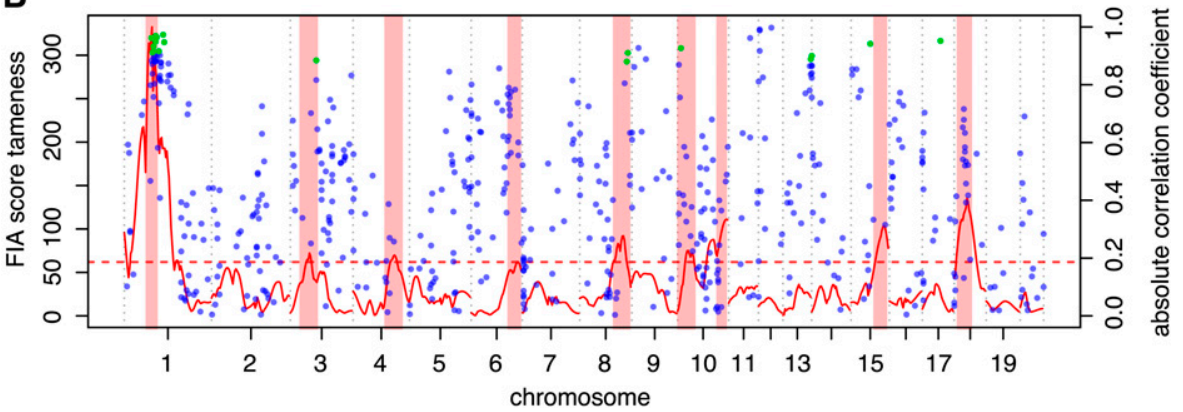

Figure 5 Correlations between allele effects of eQTL and tameness QTL along the genome. Green, significant $(P<0.05$, Benjamini-Hochberg corrected) correlations; blue, nonsignificant correlations. FIA mapping scores for tameness are shown as the solid red line and the confidence intervals for significant tameness QTL are shown as light red rectangles. Red dashed horizontal line, 2\% FDR score threshold for tameness QTL. (A) Local eQTL that overlap a tameness QTL tested at the peak of the corresponding tameness QTL. (B) Allele effect correlations at all local eQTL peak positions. Note that 18 of 23 significant allele effect correlations occur at local eQTL that overlap with a tameness QTL. interactions, where the effect of one locus depends on the genotype of another locus (Phillips 2008) may also play a role, especially given that we have earlier detected epistatic interactions between tameness QTL (Albert et al. 2009). The large number of genes and pathways described in the literature influencing behavioral traits related to tameness such as anxiety and aggression makes it plausible that genes in multiple biological pathways could have reacted to the strong selection in these rats (Hovatta and Barlow 2008; Le-Niculescu et al. 2011).

A major goal of our analyses was to compare genetic influences on brain gene expression to those on tameness and aggression. Genetic influences on brain gene expression are plausible contributors to behavioral phenotypes. We found numerous eQTL that overlapped the eight tameness QTL (Table S4). In particular, the strongest tameness QTL (Tame-1) contained multiple highly significant eQTL with allele effects that were correlated with those on tameness. This result indicates that Tame-1 might harbor several genes influencing behavior through altered gene expression in the brain. QTL identified in $\mathrm{F}_{2}$ crosses frequently fractionate into loci of more minor effects during fine mapping (Demarest et al. 2001; Mozhui et al. 2008) and contain multiple causative variants (Baud et al. 2013; Flister et al. 2013). An advanced intercross using large numbers of rats to generate more recombination events will be necessary to investigate whether variation at more than one gene underlies Tame-1 (Darvasi and Soller 1995).

We utilized the ability of FIA to infer the effects of multiple alleles at both tameness QTL and eQTL to prioritize potentially causative genes among genes with eQTL that overlap a tameness locus. The rationale is that if multiple haplotypes of a gene at an overlapping QTL/eQTL locus influence both tameness and the expression of a gene, the relative strengths of their allele effects on tameness and expression should be correlated. In contrast, if the QTL/eQTL locus is due to different variants affecting tameness and gene expression in the brain, it is less likely that their effects are correlated. Thus, identification of pairs of eQTL and tameness QTL with segregating alleles and correlated genetic effects allows us to select more likely causal genes (Nica et al. 2010). For example, of 43 eQTL that overlap Tame-1, only 10 had significant allele effect correlations in both our scans, implying these $23 \%$ of the genes as more likely to be causal. This type of prioritization would not have been possible in a cross between two inbred lines.

In addition to correlation of allele effects on behavior and gene expression, we considered differential brain gene expression between the tame and aggressive rat lines and correlation of gene expression traits with tameness across $\mathrm{F}_{2}$ animals to identify the most plausible candidate causal genes. The genes highlighted by these analyses (Figure 6) include Lgi4, Gltscr2, Zfp40, and Slc17a7, all of which are located in Tame-1 and had significant eQTL allele effect correlations with this tameness QTL. With the exception of Slc17a7, none of these genes have to date been implicated in the regulation of a behavioral trait.

Lgi4 is involved in glial cell proliferation and axon myelination in the peripheral nervous system (Kegel et al. 2013). There is also evidence for a biological role in the central nervous system: polymorphisms in Lgi4 have been associated with central epilepsy conditions (Gu et al. 2004; Ishii et al. 2010). We found Lgi4 to be differentially expressed between the tame and aggressive selection lines. Alleles from the tame line decreased Lgi4 expression both in the contrast between the selected lines and in the $\mathrm{F}_{2}$ population.

Gltscr2 encodes a regulator of the tumor suppressor genes p53 (Golomb and Oren 2011) and PTEN (Yim et al. 2007) 


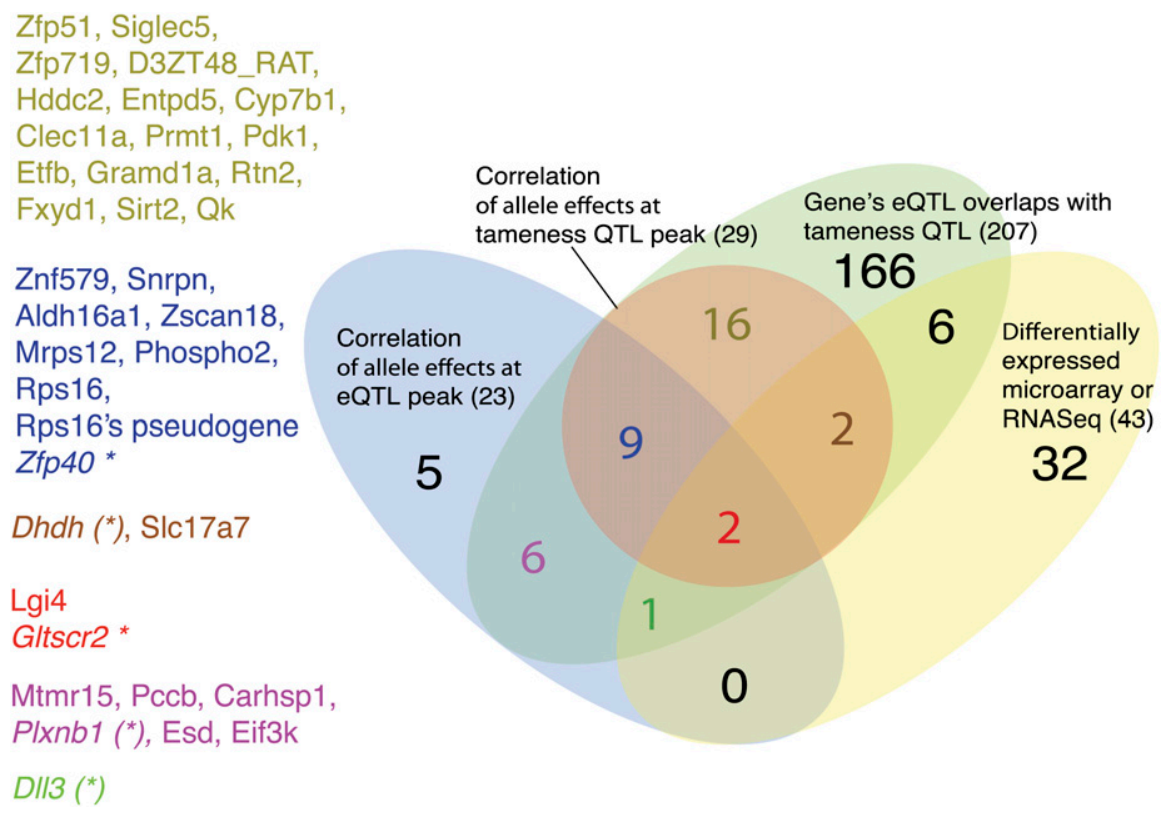

Zfp51, Siglec5,

Zfp719, D3ZT48_RAT,

Hddc2, Entpd5, Cyp7b1,

Clec11a, Prmt1, Pdk1,

Etfb, Gramd1a, Rtn2,

Znf579, Snrpn,

Aldh16a1, Zscan18,

Mrps12, Phospho2,

Rps16,

Rps16's pseudogene

Zfp40 *

of allele effects

oQTL peak (23)

\section{5}

of allele effects

(29)

166

Figure 6 Genes that show evidence for influencing tameness and aggression. Shown are the number and names of genes that meet at least one of five criteria for involvement in tameness: (i) positional overlap between eQTL and tameness QTL (green oval); significant allele effect correlations with tameness QTL at either (ii) eQTL peaks (blue oval) or (iii) tameness QTL peaks (red circle); (iv) differential expression in RNA-seq frontal cortex or in whole brain microarray between the tame and aggressive populations (yellow oval); (v) significant correlation with tameness in $F_{2}$ animals [gene names in italics, * indicates significance across all genes, and (*) indicates significance only for genes whose eQTL overlap tameness QTL]. The numbers in parentheses indicate the number of genes that fulfilled the respective criterion. Gene names given on the left are color coded corresponding to those in the figure and are based on the criteria the given gene matches. Note that the overlap between the two scans for allele effect correlations is based on strict significance criteria in both scans (Materials and Methods), resulting in a conservative estimate of the intersection. that shows lower expression in several tumors including gliomas (Kim et al. 2008). It was also described to have functions in embryogenesis and embryonic stem cell survival (Sasaki et al. 2011). Alleles from the tame line increased Gltscr 2 expression both when comparing the two selection lines and in the $\mathrm{F}_{2}$ population. In addition, Gltscr2 expression was positively correlated with tameness across the $\mathrm{F}_{2}$ animals.

Zfp40 (Zinc finger protein 40) is a little-studied gene that it is expressed in murine embryonic nervous system development (Noce et al. 1993). In the $F_{2}$ animals, alleles from the tame line increased Zfp 40 expression and the expression of $Z f p 40$ was significantly and positively correlated with tameness.

Slc17a7 belongs to a family of vesicular glutamate transporter genes and has been previously implicated in the regulation of behavior. Mice that carry a hemizygous knockout such that they express only half the amount of Slc17a7 compared to wild-type mice, are more anxious (Tordera et al. 2007) and more vulnerable to chronic mild stress (GarciaGarcia et al. 2009; Farley et al. 2012). Here, the expression of Slc17a7 in brain hemispheres in rats from the tame selection line was twice that in the aggressive rats. However, the eQTL acted in the opposite direction: alleles from the aggressive founder animals increase Slc17a7 expression. Further, Slc17a7 expression does not correlate with tameness in the $\mathrm{F}_{2}$ animals, and Slc17a7 is located at some distance $(13 \mathrm{cM})$ from the peak of Tame-1. Thus, although it is a promising candidate gene, precisely how Slc17a7 may contribute to tameness and aggression remains unclear.

We found several genes that were not themselves located in tameness QTL, but whose expression was influenced by distant eQTL that did overlap tameness QTL. Trans-acting variants in the tameness QTL might lead to differential expression of these genes, which in turn might shape the physiological pathways that regulate tame or aggressive behavior.
An example of such a gene is Cyp7b1, whose expression is influenced by an eQTL that overlaps a tameness QTL on chromosome six. Cyp7b1 encodes a 25-hydroxycholesterol 7-alpha-hydroxylase that is involved in DHEA metabolism (Yau et al. 2003). Interestingly, a key enzyme involved in the metabolism of this neurosteroid is located in a fixed duplication in 20 tame founder rats in Tame-1 (A. Cagan, personal communication). It is interesting that genetic variation in two independent tameness QTL is connected to the DHEA system, making this system an interesting candidate for further studies into the regulation of tameness and aggression.

The gene Htr $3 a$ encodes a subunit of the ionotropic serotonin receptor type 3 (5-HT3). It has earlier been shown that there are differences in serotonin level between the tame and aggressive founder lines in different brain regions (Naumenko et al. 1989; Albert et al. 2008). Further, serotonin and its metabolite were higher in several brain regions in a line of foxes selected for tameness than in foxes not selected for behavior (Popova et al. 1976, 1991). The 5-HT3 receptor density is also greater in hamsters showing impulsive aggression (Cervantes and Delville 2009) and Htr3a knockout mice exhibit changes in anxiety-like behavior and hypothalamus-pituitary- adrenal (HPA) axis response to stress (Bhatnagar et al. 2004). In this study, Htr3a is differentially expressed between the tame and aggressive founders at a 10\% FDR and weakly correlated (but not robust to multiple testing) with tameness in the $\mathrm{F}_{2}$. Also, the expression of the gene Htr3a is influenced by two distant eQTL, one of which overlaps with the second strongest FIA tameness QTL on chromosome 18. The allele effect correlations at this tameness QTL were nominally significant (but not robust to multiple testing). Although the evidence for Htr3a is marginal in each independent analysis, the coherency in the results indicates that it is a useful gene for further studies into the basis of tameness in this population. 
In summary, we identified hundreds of genomic loci that influence gene expression levels in the brains of rats that had been bred for tame and aggressive behavior toward humans. Differences in brain gene expression are an attractive avenue by which genetic differences between and within these two rat lines may contribute to the strong differences in behavior. We also found six loci with effects on tameness that had been missed by earlier work, illustrating that the genetic architecture of behavior is complex in this artificially selected population. The expression of several genes inside the strongest tameness QTL was influenced by local eQTL with very strong effects. These genes are promising candidates for functional followup work to study if and how they contribute to tameness and aggression.

\section{Acknowledgments}

We are grateful to Martin Kircher, Martin Kuhlwilm, Michael Dannemann, Rigo Schultz, Udo Stenzel, and other past and present members of the Max Planck Institute for Evolutionary Anthropology for help with bioinformatic analyses. We thank Dominic Wright and one anonymous reviewer for their helpful comments on the manuscript. This work was supported by the Max Planck Society, an Sfb 1052 German research foundation (DFG) and Integrated Research and Treatment Center (IFB) AdiposityDiseases Leipzig German Federal Ministry of Education and Research (BMBF) to T.S., a DFG research fellowship AL 1525/1-1 to F.W.A., a Studienstiftung des deutschen Volkes stipend to H.O.H., and MD1 and MD2 (IFB AdiposityDiseases Leipzig) stipends to H.O.H.

\section{Literature Cited}

Albert, F. W., O. Shchepina, C. Winter, H. Römpler, D. Teupser et al., 2008 Phenotypic differences in behavior, physiology and neurochemistry between rats selected for tameness and for defensive aggression towards humans. Horm. Behav. 53: 413-421.

Albert, F. W., O. Carlborg, I. Plyusnina, F. Besnier, D. Hedwig et al., 2009 Genetic architecture of tameness in a rat model of animal domestication. Genetics 182: 541-554.

Albert, F. W., E. Hodges, J. D. Jensen, F. Besnier, Z. Xuan et al., 2011 Targeted resequencing of a genomic region influencing tameness and aggression reveals multiple signals of positive selection. Heredity 107: 205-214.

Albert, F. W., M. Somel, M. Carneiro, A. Aximu-Petri, M. Halbwax et al., 2012 A comparison of brain gene expression levels in domesticated and wild animals. PLoS Genet. 8: e1002962.

Baud, A., R. Hermsen, V. Guryev, P. Stridh, D. Graham et al., 2013 Combined sequence-based and genetic mapping analysis of complex traits in outbred rats. Nat. Genet. 45: 767-775.

Belyaev, D. K., 1979 The Wilhelmine E. Key 1978 invitational lecture. Destabilizing selection as a factor in domestication. J. Hered. 70: 301-308.

Bendesky, A., J. Pitts, M. V. Rockman, W. C. Chen, M. W. Tan et al., 2012 Long-range regulatory polymorphisms affecting a GABA receptor constitute a quantitative trait locus (QTL) for social behavior in Caenorhabditis elegans. PLoS Genet. 8: e1003157.
Benjamini, Y., and D. Yekutieli, 2005 Quantitative trait Loci analysis using the false discovery rate. Genetics 171: 783-790.

Besnier, F., and O. Carlborg, 2007 A general and efficient method for estimating continuous IBD functions for use in genome scans for QTL. BMC Bioinformatics 8: 440.

Besnier, F., and O. Carlborg, 2009 A genetic algorithm based method for stringent haplotyping of family data. BMC Genet. 10: 57.

Bhatnagar, S., L. M. Sun, J. Raber, S. Maren, D. Julius et al., 2004 Changes in anxiety-related behaviors and hypothalamicpituitary-adrenal activity in mice lacking the 5-HT-3A receptor. Physiol. Behav. 81: 545-555.

Bolstad, B. M., R. A. Irizarry, M. Astrand, and T. P. Speed, 2003 A comparison of normalization methods for high density oligonucleotide array data based on variance and bias. Bioinformatics 19: 185193.

Broman, K. W., and S. Saunak, 2009 A Guide to QTL Mapping with $R / q t l$, Springer-Verlag, Berlin.

Broman, K. W., H. Wu, S. Sen, and G. A. Churchill, 2003 R/qtl: QTL mapping in experimental crosses. Bioinformatics 19: 889-890.

Burke, M. K., J. P. Dunham, P. Shahrestani, K. R. Thornton, M. R. Rose et al., 2010 Genome-wide analysis of a long-term evolution experiment with Drosophila. Nature 467: 587-590.

Calboli, F. C., F. Tozzi, N. W. Galwey, A. Antoniades, V. Mooser et al., 2010 A genome-wide association study of neuroticism in a population-based sample. PLoS ONE 5: e11504.

Cervantes, M. C., and Y. Delville, 2009 Serotonin 5-HT1A and 5HT3 receptors in an impulsive-aggressive phenotype. Behav. Neurosci. 123: 589-598.

Dai, M., P. Wang, A. D. Boyd, G. Kostov, B. Athey et al., 2005 Evolving gene/transcript definitions significantly alter the interpretation of GeneChip data. Nucleic Acids Res. 33: e175.

Darvasi, A., and M. Soller, 1995 Advanced intercross lines, an experimental population for fine genetic mapping. Genetics 141: 1199-1207.

de Jong, S., M. J. H. Kas, J. Kiernan, A. G. de Mooij-van Malsen, H. Oppelaar et al., 2011 Hippocampal gene expression analysis highlights Ly6a/Sca-1 as candidate gene for previously mapped novelty induced behaviors in mice. PLoS ONE 6: e20716.

Deary, I. J., W. Johnson, and L. M. Houlihan, 2009 Genetic foundations of human intelligence. Hum. Genet. 126: 215-232.

Demarest, K., J. Koyner, J. McCaughran, Jr., L. Cipp, and R. Hitzemann, 2001 Further characterization and high-resolution mapping of quantitative trait loci for ethanol-induced locomotor activity. Behav. Genet. 31: 79-91.

Diamond, J., 1999 Guns, Germs, and Steel: The Fates of Human Societies, Chatto \& Windus, London.

Farley, S., S. Dumas, S. El Mestikawy, and B. Giros, 2012 Increased expression of the Vesicular Glutamate Transporter-1 (VGLUT1) in the prefrontal cortex correlates with differential vulnerability to chronic stress in various mouse strains: effects of fluoxetine and MK-801. Neuropharmacology 62: 503-517.

Flint, J., 2003 Analysis of quantitative trait loci that influence animal behavior. J. Neurobiol. 54: 46-77.

Flint, J., and M. R. Munafo, 2013 Candidate and non-candidate genes in behavior genetics. Curr. Opin. Neurobiol. 23: 57-61.

Flister, M. J., S. W. Tsaih, C. C. O'Meara, B. Endres, M. J. Hoffman et al., 2013 Identifying multiple causative genes at a single GWAS locus. Genome Res. 23: 1996-2002.

Garcia-Garcia, A. L., N. Elizalde, D. Matrov, J. Harro, S. M. Wojcik et al., 2009 Increased vulnerability to depressive-like behavior of mice with decreased expression of VGLUT1. Biol. Psychiatry 66: 275-282.

Golomb, L., and M. Oren, 2011 DePICTing p53 activation: a new nucleolar link to cancer. Cancer Cell 20: 283-284.

Goodson, M., M. B. Rust, W. Witke, D. Bannerman, R. Mott et al., 2012 Cofilin-1: a modulator of anxiety in mice. PLoS Genet. 8: e1002970. 
Gu, W., T. Sander, T. Becker, and O. K. Steinlein, 2004 Genotypic association of exonic LGI4 polymorphisms and childhood absence epilepsy. Neurogenetics 5: 41-44.

Haley, C. S., and S. A. Knott, 1992 A simple regression method for mapping quantitative trait loci in line crosses using flanking markers. Heredity 69: 315-324.

Heinig, M., E. Petretto, C. Wallace, L. Bottolo, M. Rotival et al., 2010 A trans-acting locus regulates an anti-viral expression network and type 1 diabetes risk. Nature 467: 460-464.

Hitzemann, R., C. Reed, B. Malmanger, M. Lawler, B. Hitzemann et al., 2004 On the integration of alcohol-related quantitative trait loci and gene expression analyses. Alcohol. Clin. Exp. Res. 28: $1437-1448$.

Hovatta, I., and C. Barlow, 2008 Molecular genetics of anxiety in mice and men. Ann. Med. 40: 92-109.

Huang, D. W., B. T. Sherman, and R. A. Lempicki, 2008 Systematic and integrative analysis of large gene lists using DAVID bioinformatics resources. Nat. Protoc. 4: 44-57.

Hubner, N., C. A. Wallace, H. Zimdahl, E. Petretto, H. Schulz et al., 2005 Integrated transcriptional profiling and linkage analysis for identification of genes underlying disease. Nat. Genet. 37: 243-253.

Hunt, K. A., V. Mistry, N. A. Bockett, T. Ahmad, M. Ban et al., 2013 Negligible impact of rare autoimmune-locus coding-region variants on missing heritability. Nature 498: 232-235.

Irizarry, R. A., B. Hobbs, F. Collin, Y. D. Beazer-Barclay, K. J. Antonellis et al., 2003 Exploration, normalization, and summaries of high density oligonucleotide array probe level data. Biostatistics 4: 249-264.

Ishii, A., B. Zhang, S. Kaneko, and S. Hirose, 2010 Positive association between benign familial infantile convulsions and LGI4. Brain Dev. 32: 538-543.

Johansson, A. M., M. E. Pettersson, P. B. Siegel, and O. Carlborg, 2010 Genome-wide effects of long-term divergent selection. PLoS Genet. 6: e1001188.

Kegel, L., E. Aunin, D. N. Meijer, and J. R. Bermingham, Jr.., 2013 LGI proteins in the nervous system. ASN Neuro 5: 167-181.

Kelly, S. A., D. L. Nehrenberg, K. Hua, T. Garland, Jr., and D. Pomp, 2012 Functional genomic architecture of predisposition to voluntary exercise in mice: expression QTL in the brain. Genetics 191: 643-654.

Kim, Y. J., Y. E. Cho, Y. W. Kim, J. Y. Kim, S. Lee et al., 2008 Suppression of putative tumour suppressor gene GLTSCR2 expression in human glioblastomas. J. Pathol. 216: 218-224.

Kircher, M., U. Stenzel, and J. Kelso, 2009 Improved base calling for the Illumina Genome Analyzer using machine learning strategies. Genome Biol. 10: R83.

Kuhlwilm, M., A. Davierwala, and S. Paabo, 2013 Identification of putative target genes of the transcription factor RUNX2. PLoS ONE 8: e83218.

Langfelder, P., and S. Horvath, 2008 WGCNA: an R package for weighted correlation network analysis. BMC Bioinformatics 9: 559.

Langley, S. R., L. Bottolo, J. Kunes, J. Zicha, V. Zidek et al., 2013 Systems-level approaches reveal conservation of transregulated genes in the rat and genetic determinants of blood pressure in humans. Cardiovasc. Res. 97: 653-665.

Laurie, C. C., S. D. Chasalow, J. R. LeDeaux, R. McCarroll, D. Bush et al., 2004 The genetic architecture of response to long-term artificial selection for oil concentration in the maize kernel. Genetics 168: 2141-2155.

Le-Niculescu, H., Y. Balaraman, S. D. Patel, M. Ayalew, J. Gupta et al., 2011 Convergent functional genomics of anxiety disorders: translational identification of genes, biomarkers, pathways and mechanisms. Transl. Psychiatry 1: e9.
Leek, J. T., and J. D. Storey, 2007 Capturing heterogeneity in gene expression studies by surrogate variable analysis. PLoS Genet. 3: 1724-1735.

Leek, J. T., R. B. Scharpf, H. C. Bravo, D. Simcha, B. Langmead et al., 2010 Tackling the widespread and critical impact of batch effects in high-throughput data. Nat. Rev. Genet. 11: 733-739.

Leek, J. T., W. E. Johnson, H. S. Parker, A. E. Jaffe, and J. D. Storey, 2012 The sva package for removing batch effects and other unwanted variation in high-throughput experiments. Bioinformatics 28: 882-883.

Lynch, M., and B. Walsh, 1998 Genetics and Analysis of Quantitative Traits, Sinauer Associates, Sunderland, MA.

Mackay, T. F. C., E. A. Stone, and J. F. Ayroles, 2009 The genetics of quantitative traits: challenges and prospects. Nat. Rev. Genet. 10: 565-577.

Manichaikul, A., J. Dupuis, S. Sen, and K. W. Broman, 2006 Poor performance of bootstrap confidence intervals for the location of a quantitative trait locus. Genetics 174: 481-489.

McGrath, P. T., M. V. Rockman, M. Zimmer, H. Jang, E. Z. Macosko et al., 2009 Quantitative mapping of a digenic behavioral trait implicates globin variation in C. elegans sensory behaviors. Neuron 61: 692-699.

Mehrabian, M., H. Allayee, J. Stockton, P. Y. Lum, T. A. Drake et al., 2005 Integrating genotypic and expression data in a segregating mouse population to identify 5-lipoxygenase as a susceptibility gene for obesity and bone traits. Nat. Genet. 37: 1224-1233.

Moffatt, M. F., M. Kabesch, L. Liang, A. L. Dixon, D. Strachan et al., 2007 Genetic variants regulating ORMDL3 expression contribute to the risk of childhood asthma. Nature 448: 470-473.

Mozhui, K., D. C. Ciobanu, T. Schikorski, X. Wang, L. Lu et al., 2008 Dissection of a QTL hotspot on mouse distal chromosome 1 that modulates neurobehavioral phenotypes and gene expression. PLoS Genet. 4: e1000260.

Musunuru, K., A. Strong, M. Frank-Kamenetsky, N. E. Lee, T. Ahfeldt et al., 2010 From noncoding variant to phenotype via SORT1 at the 1p13 cholesterol locus. Nature 466: 714-719.

Naumenko, E. V., N. K. Popova, E. M. Nikulina, N. N. Dygalo, G. T. Shishkina et al., 1989 Behavior, adrenocortical activity, and brain monoamines in Norway rats selected for reduced aggressiveness towards man. Pharmacol. Biochem. Behav. 33: 85-91.

Nelson, R. M., X. Shen, and O. Carlborg, 2011 qtl.outbred: interfacing outbred line cross data with the R/qtl mapping software. BMC Res. Notes 4: 154.

Nica, A. C., S. B. Montgomery, A. S. Dimas, B. E. Stranger, C. Beazley et al., 2010 Candidate causal regulatory effects by integration of expression QTLs with complex trait genetic associations. PLoS Genet. 6: e1000895.

Noce, T., Y. Fujiwara, M. Ito, T. Takeuchi, N. Hashimoto et al., 1993 A novel murine zinc finger gene mapped within the tw18 deletion region expresses in germ cells and embryonic nervous system. Dev. Biol. 155: 409-422.

Otowa, T., E. Yoshida, N. Sugaya, S. Yasuda, Y. Nishimura et al., 2009 Genome-wide association study of panic disorder in the Japanese population. J. Hum. Genet. 54: 122-126.

Perlstein, E. O., D. M. Ruderfer, D. C. Roberts, S. L. Schreiber, and L. Kruglyak, 2007 Genetic basis of individual differences in the response to small-molecule drugs in yeast. Nat. Genet. 39: 496-502.

Petretto, E., J. Mangion, N. J. Dickens, S. A. Cook, M. K. Kumaran et al., 2006a Heritability and tissue specificity of expression quantitative trait loci. PLoS Genet. 2: e172.

Petretto, E., J. Mangion, M. Pravanec, N. Hubner, and T. J. Aitman, 2006b Integrated gene expression profiling and linkage analysis in the rat. Mamm. Genome 17: 480-489.

Pettersson, M. E., A. M. Johansson, P. B. Siegel, and O. Carlborg, 2013 Dynamics of adaptive alleles in divergently selected body weight lines of chickens. G3 (Bethesda) 3: 2305-2312. 
Phillips, P. C., 2008 Epistasis: the essential role of gene interactions in the structure and evolution of genetic systems. Nat. Rev. Genet. 9: 855-867.

Plyusnina, I., and I. Oskina, 1997 Behavioral and adrenocortical responses to open-field test in rats selected for reduced aggressiveness toward humans. Physiol. Behav. 61: 381-385.

Plyusnina, I. Z., I. N. Oskina, M. A. Tibeikina, and N. K. Popova, 2009 Cross-fostering effects on weight, exploratory activity, acoustic startle reflex and corticosterone stress response in Norway gray rats selected for elimination and for enhancement of aggressiveness towards human. Behav. Genet. 39: 202-212.

Pong-Wong, R., A. W. George, J. A. Woolliams, and C. S. Haley, 2001 A simple and rapid method for calculating identity-by-descent matrices using multiple markers. Genet. Sel. Evol. 33: 453-471.

Popova, N. K., N. N. Voitenko, and L. N. Trut, 1976 Changes in the content of serotonin and 5-hydroxyindoleacetic acid in the brain in the selection of silver foxes according to behavior. Neurosci. Behav. Physiol. 7: 72-74.

Popova, N. K., N. N. Voitenko, A. V. Kulikov, and D. F. Avgustinovich, 1991 Evidence for the involvement of central serotonin in mechanism of domestication of silver foxes. Pharmacol. Biochem. Behav. 40: 751-756.

R Development Core Team, 2012 R: a Language and Environment for Statistical Computing. R Foundation for Statistical Computing, Vienna.

Rietveld, C. A., S. E. Medland, J. Derringer, J. Yang, T. Esko et al., 2013 GWAS of 126,559 individuals identifies genetic variants associated with educational attainment. Science 340: 1467-1471.

Roberts, A., C. Trapnell, J. Donaghey, J. L. Rinn, and L. Pachter, 2011 Improving RNA-Seq expression estimates by correcting for fragment bias. Genome Biol. 12: R22.

Roider, H. G., T. Manke, S. O'Keeffe, M. Vingron, and S. A. Haas, 2009 PASTAA: identifying transcription factors associated with sets of co-regulated genes. Bioinformatics 25: 435-442.

Rönnegård, L., F. Besnier, and O. Carlborg, 2008 An improved method for quantitative trait loci detection and identification of within-line segregation in F2 intercross designs. Genetics 178: 2315-2326.

Saba, L. M., B. Bennett, P. L. Hoffman, K. Barcomb, T. Ishii et al., 2011 A systems genetic analysis of alcohol drinking by mice, rats and men: influence of brain GABAergic transmission. Neuropharmacology 60: 1269-1280.

Sasaki, M., K. Kawahara, M. Nishio, K. Mimori, R. Kogo et al., 2011 Regulation of the MDM2-P53 pathway and tumor growth by PICT1 via nucleolar RPL11. Nat. Med. 17: 944-951.

Schadt, E. E., J. Lamb, X. Yang, J. Zhu, S. Edwards et al., 2005 An integrative genomics approach to infer causal associations between gene expression and disease. Nat. Genet. 37: 710-717.

Schroeder, A., O. Mueller, S. Stocker, R. Salowsky, M. Leiber et al., 2006 The RIN: an RNA integrity number for assigning integrity values to RNA measurements. BMC Mol. Biol. 7: 3.
Sokolowska, E., and I. Hovatta, 2013 Anxiety genetics: findings from cross-species genome-wide approaches. Biol Mood Anxiety Disord 3: 9.

Terracciano, A., S. Sanna, M. Uda, B. Deiana, G. Usala et al., 2010 Genome-wide association scan for five major dimensions of personality. Mol. Psychiatry 15: 647-656.

Tordera, R. M., S. Totterdell, S. M. Wojcik, N. Brose, N. Elizalde et al., 2007 Enhanced anxiety, depressive-like behaviour and impaired recognition memory in mice with reduced expression of the vesicular glutamate transporter 1 (VGLUT1). Eur. J. Neurosci. 25: 281-290.

Trapnell, C., L. Pachter, and S. L. Salzberg, 2009 TopHat: discovering splice junctions with RNA-Seq. Bioinformatics 25: 1105-1111.

Trapnell, C., B. A. Williams, G. Pertea, A. Mortazavi, G. Kwan et al., 2010 Transcript assembly and quantification by RNA-Seq reveals unannotated transcripts and isoform switching during cell differentiation. Nat. Biotechnol. 28: 511-515.

Trut, L. N., 1999 Early Canid domestication: the farm-fox experiment. Am. Sci. 87: 160-169.

van Nas, A., L. Ingram-Drake, J. S. Sinsheimer, S. S. Wang, E. E. Schadt et al., 2010 Expression quantitative trait loci: replication, tissue- and sex-specificity in mice. Genetics 185: 1059-1068.

Watanabe, A., T. Toyota, Y. Owada, T. Hayashi, Y. Iwayama et al., 2007 Fabp7 maps to a quantitative trait locus for a schizophrenia endophenotype. PLoS Biol. 5: e297.

Wiener, P., and S. Wilkinson, 2011 Deciphering the genetic basis of animal domestication. Proc. Biol. Sci. 278: 3161-3170.

Willis-Owen, S. A. G., and J. Flint, 2006 The genetic basis of emotional behaviour in mice. Eur. J. Hum. Genet. 14: 721-728.

Wright, D., R. K. Butlin, and O. Carlborg, 2006 Epistatic regulation of behavioural and morphological traits in the zebrafish (Danio rerio). Behav. Genet. 36: 914-922.

Yalcin, B., S. A. Willis-Owen, J. Fullerton, A. Meesaq, R. M. Deacon et al., 2004 Genetic dissection of a behavioral quantitative trait locus shows that Rgs2 modulates anxiety in mice. Nat. Genet. 36: 1197-1202.

Yang, J., B. Benyamin, B. P. McEvoy, S. Gordon, A. K. Henders et al., 2010 Common SNPs explain a large proportion of the heritability for human height. Nat. Genet. 42: 565-569.

Yau, J. L. W., S. Rasmuson, R. Andrew, M. Graham, J. Noble et al., 2003 Dehydroepiandrosterone 7-hydroxylase CYP7B: predominant expression in primate hippocampus and reduced expression in Alzheimer's disease. Neuroscience 121: 307-314.

Yim, J. H., Y. J. Kim, J. H. Ko, Y. E. Cho, S. M. Kim et al., 2007 The putative tumor suppressor gene GLTSCR2 induces PTEN-modulated cell death. Cell Death Differ. 14: 1872-1879.

Communicating editor: E. A. Stone 


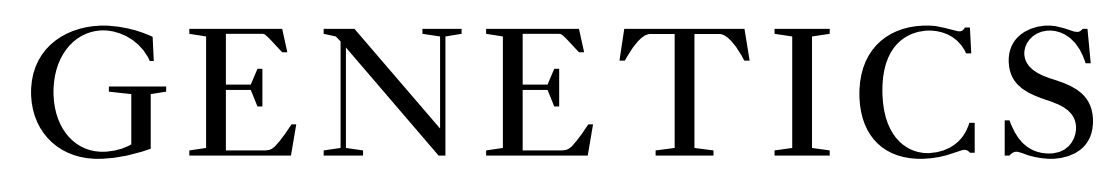

Supporting Information http://www.genetics.org/lookup/suppl/doi:10.1534/genetics.114.168948/-/DC1

\section{Genetic Influences on Brain Gene Expression in Rats Selected for Tameness and Aggression}

Henrike O. Heyne, Susann Lautenschläger, Ronald Nelson, François Besnier, Maxime Rotival, Alexander Cagan, Rimma Kozhemyakina, Irina Z. Plyusnina, Lyudmila Trut, Örjan Carlborg, Enrico Petretto, Leonid Kruglyak, Svante Pääbo, Torsten Schöneberg, and Frank W. Albert 


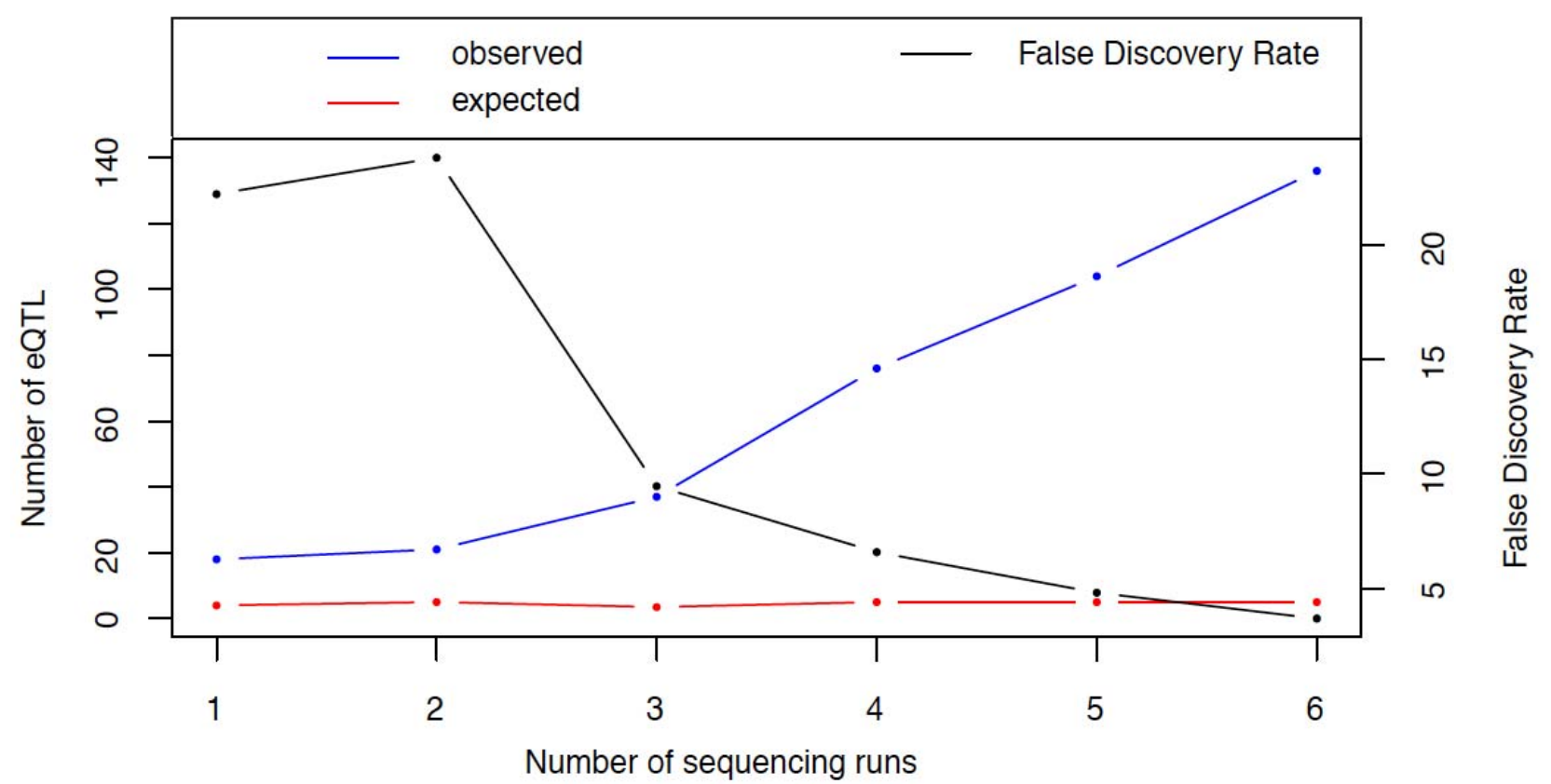

Figure S1. Expected and observed eQTL as a function of sequencing depth. These down-sampling analyses were used to evaluate the power of the study. As a proxy of sequencing depth we used the number of Illumina sequencing runs. Each Illumina sequencing run corresponded to approximately two million additional reads per animal. 


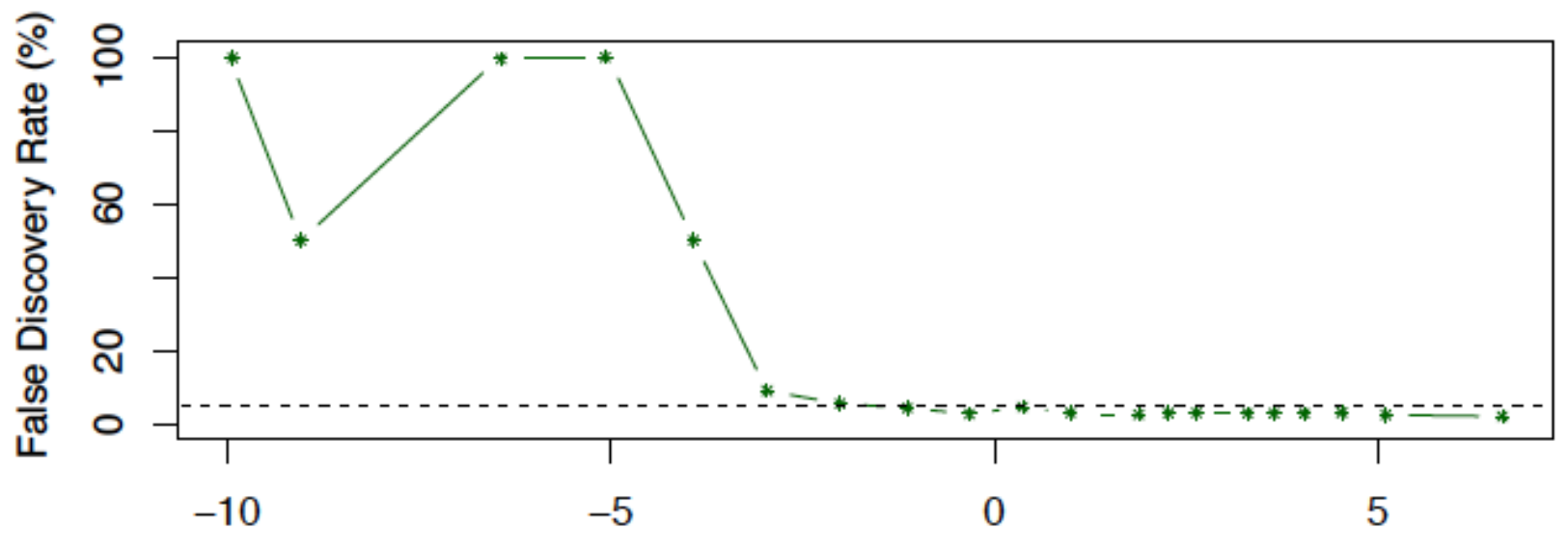

$\log 2$ mean FPKM

Figure S2. Power to detect eQTL as a function of gene expression level. Genes were grouped into 24 bins of 1,000 genes sorted by expression level. We performed eQTL mapping and permutations within each abundance bin, and plotted the resulting FDR as a function of the mean expression level within the given bin. For clarity, the four bins with the lowest abundance are not shown. The dashed horizontal line indicates the threshold of an FDR of 5\%. We used the result shown in this figure to limit eQTL mapping to genes whose expression level was high enough so that eQTL can be reliably detected. 
A
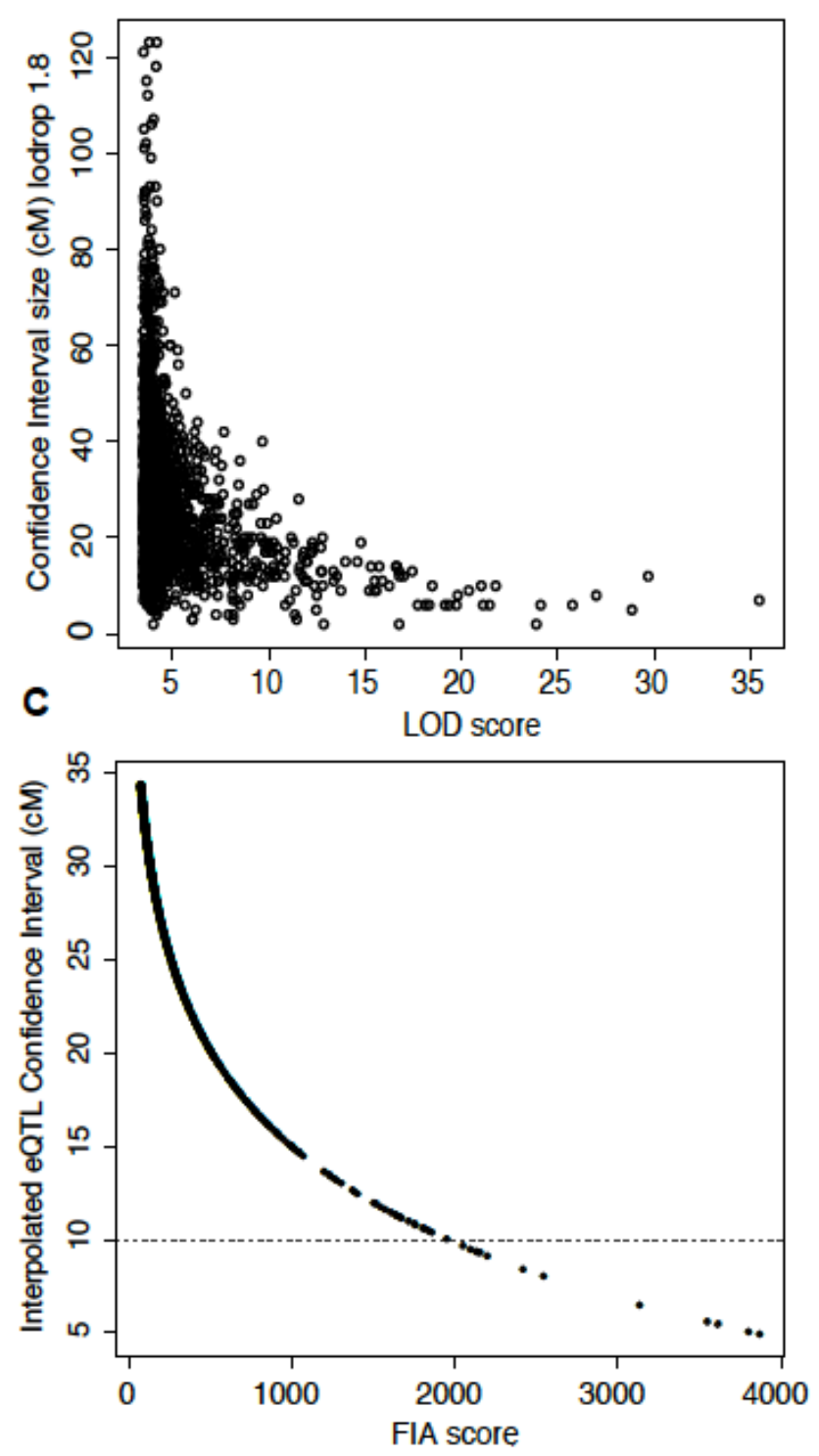

B

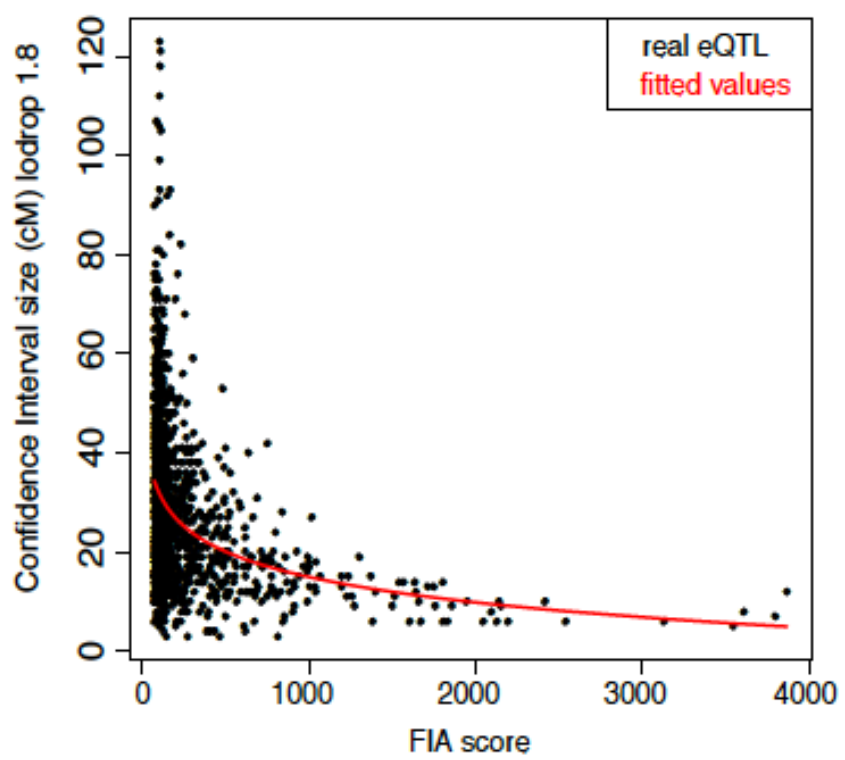

Figure S3. Defining FIA eQTL confidence intervals based on eQTL also detected by HKR. A, LOD scores of HKR eQTL and their 1.8 LOD drop confidence intervals. B, For the same set of eQTL as in A, shown are the FIA scores vs. the HKR 1.8 LOD drop confidence interval. The fitted function is shown as a red line. C, Interpolated confidence intervals for all FIA eQTL versus FIA scores. The confidence interval cut-off at $10 \mathrm{cM}$ is shown as the dashed horizontal line. 
D
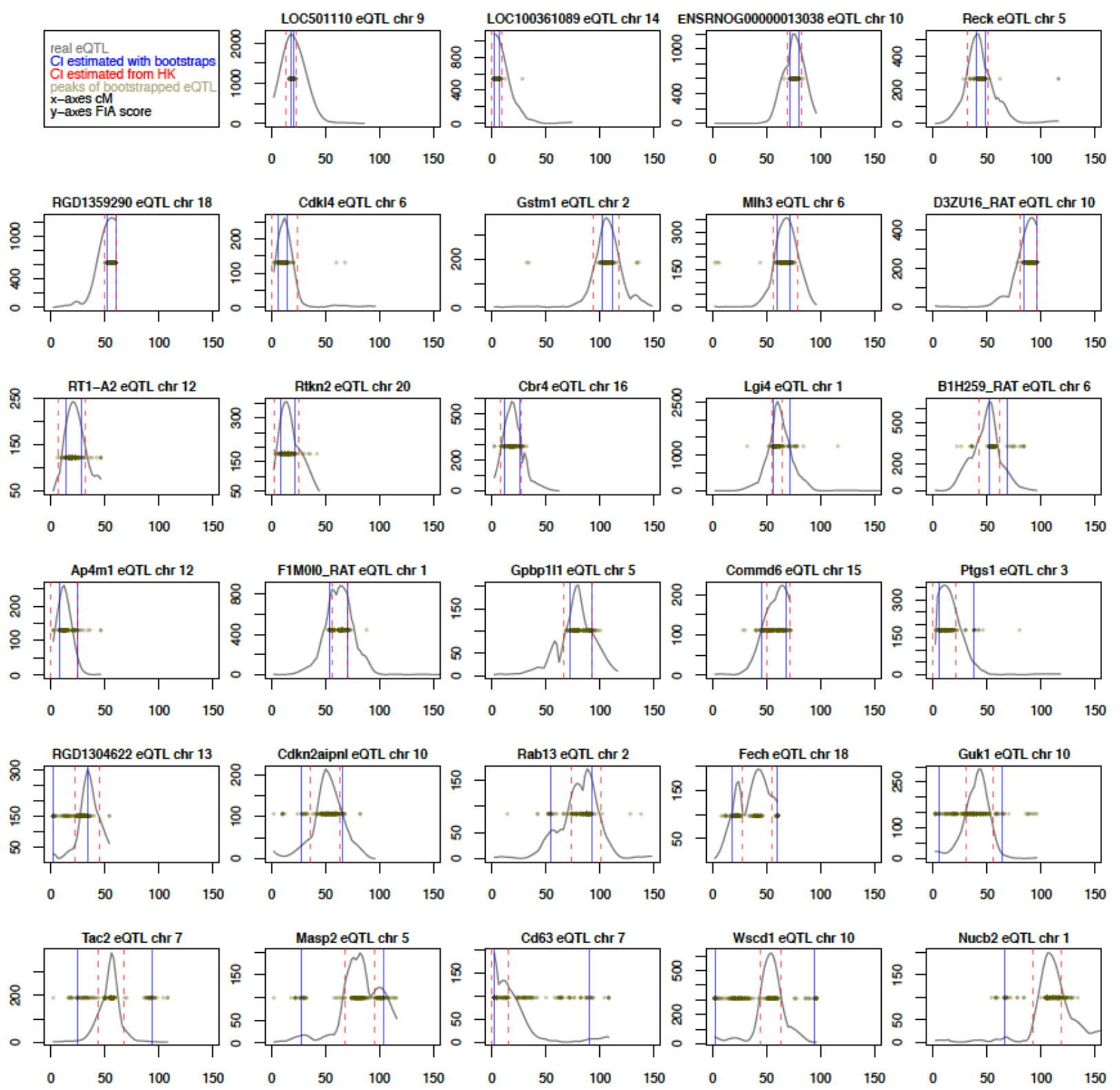

Figure S4 Comparing inferred FIA eQTL confidence intervals to bootstrap results. Shown are the 95\% respective confidence intervals for 29 randomly chosen FIA eQTL. The FIA score profiles are shown as grey lines. X-axes show genomic locations in cM. Y-axes show FIA significance scores. The peak location of each of 200 bootstraps are shown as transparent green dots. Bootstrap derived 95\% confidence intervals are shown as blue vertical lines and confidence intervals derived via HKR are shown as red dashed vertical lines. 
A
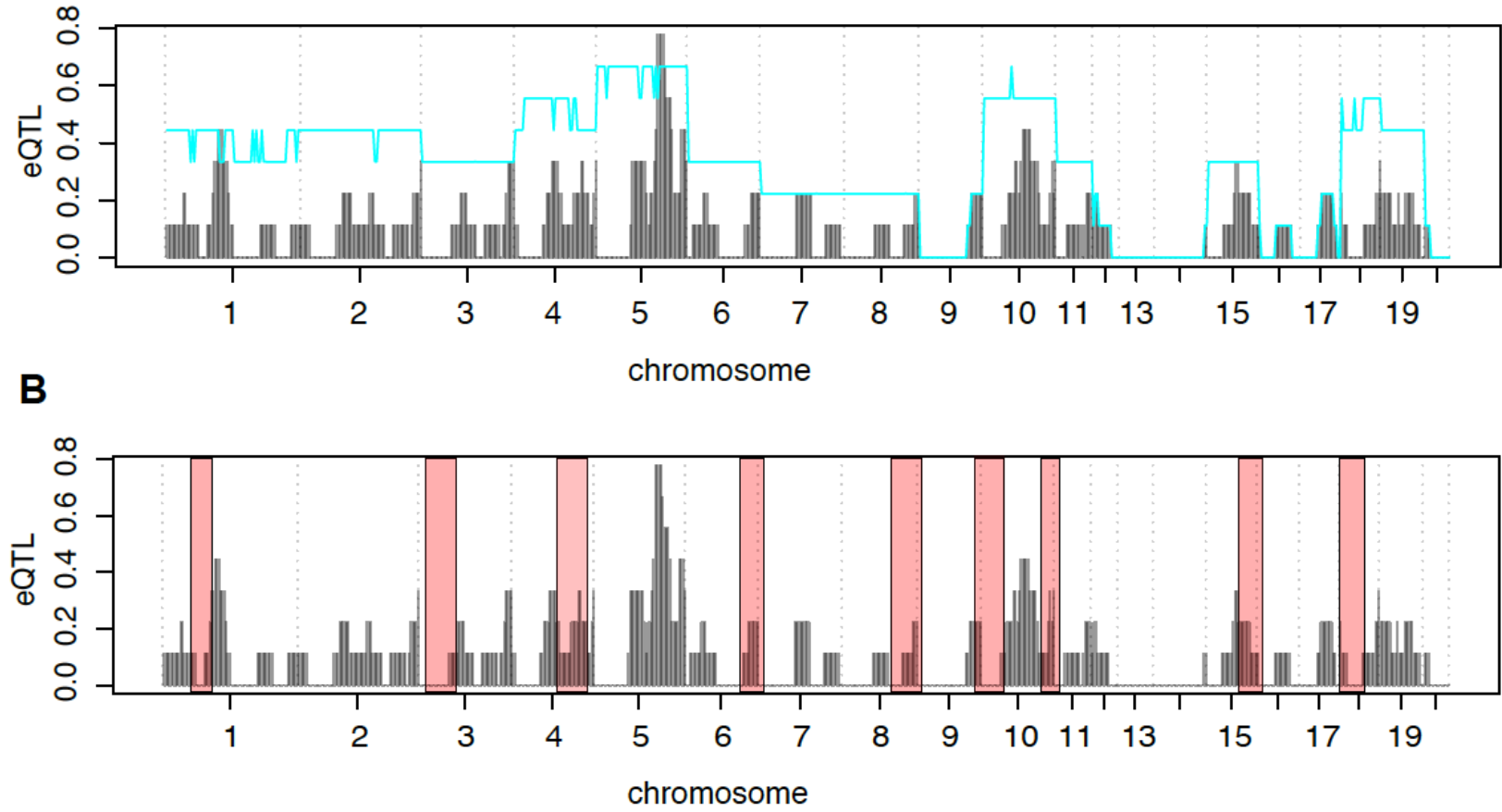

Figure S5. The number of distant eQTL along the genome. The grey bars are identical in A and B and indicate the average number of distant eQTL per 2cM window based on a sliding window analysis. The width of the window was $18 \mathrm{cM}$, corresponding to the median size of the eQTL confidence intervals. A, The $99^{\text {th }}$ quantile of the expected eQTL density from 1,000 randomizations of eQTL positions per chromosome is shown as a blue line. B, Light red rectangles: Confidence intervals for tameness QTL locations. 


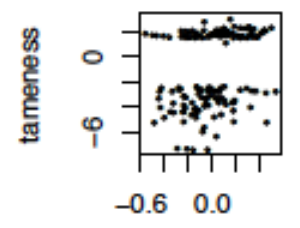

Zfp40

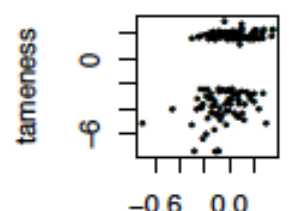

Tsen 34

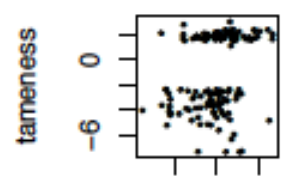

$\begin{array}{ll}-0.5 & 0.5\end{array}$

D3ZTJ9_RAT

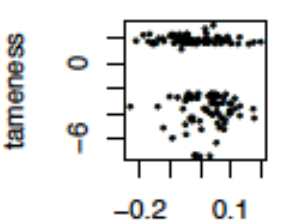

Ppt1

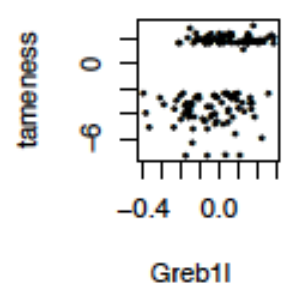

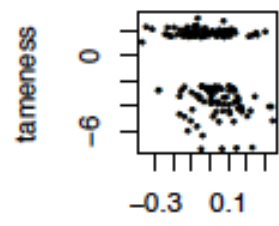

Cdk2ap1

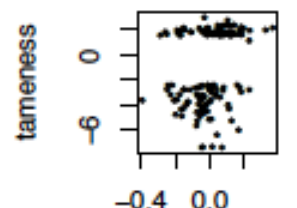

Smc4

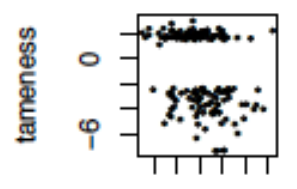

$\begin{array}{ll}-0.2 & 0.1\end{array}$

Uspl1

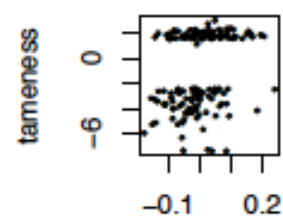

Cacna1h

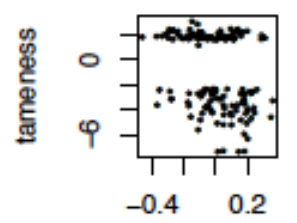

Rmi1

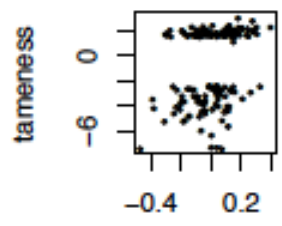

Lrrc61
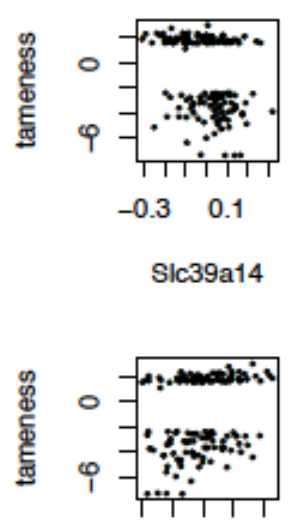

$-0.2 \quad 0.1$

Morn4

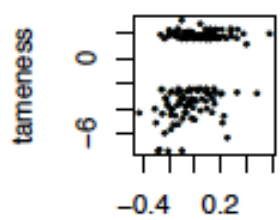

Uqcr11

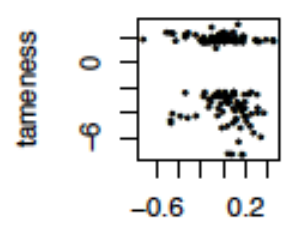

ENSRNOG00000002029

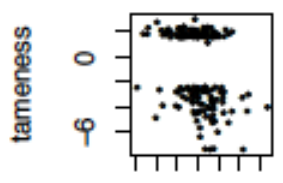

$\begin{array}{ll}-0.6 & 0.2\end{array}$

D3ZEF5_RAT
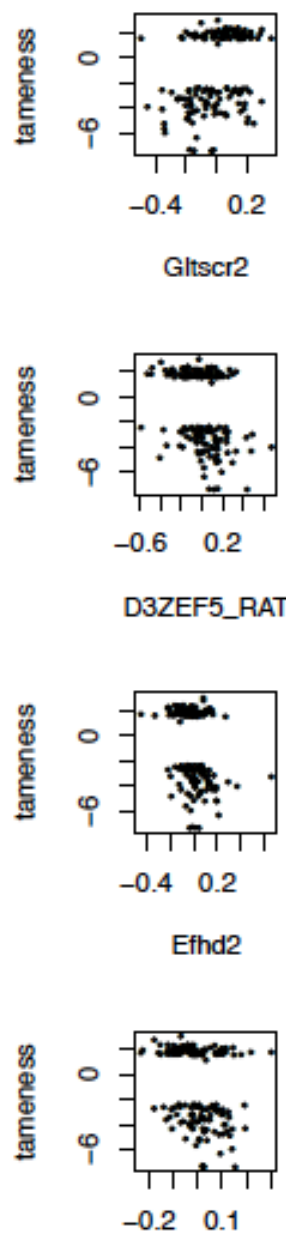

Gltscr2

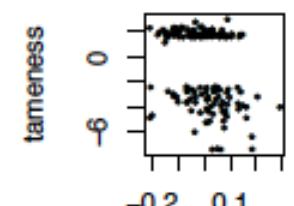

Ppp2r5c
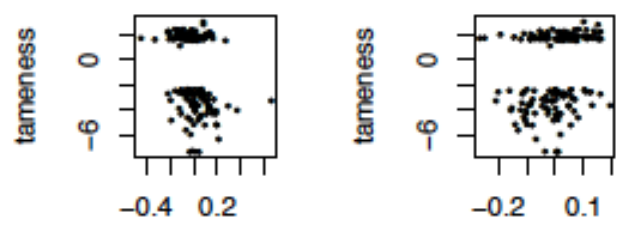

Efhd2

SQSTM1

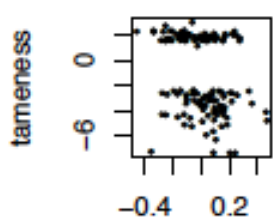

Ttpal

Pcdhga11

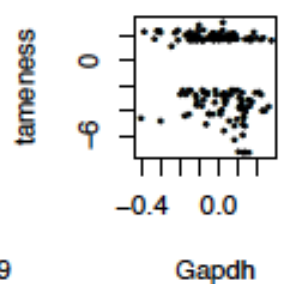

$\begin{array}{ll}-0.4 & 0.0\end{array}$

Sc4mol

Figure S6. Tameness and gene expression values for the $\mathbf{2 5}$ genes with the highest correlations between these two traits. The bimodality in the tameness score is a result of the selection of the 150 most extreme tame and aggressive individuals of the F2 intercross for inclusion in this study. 


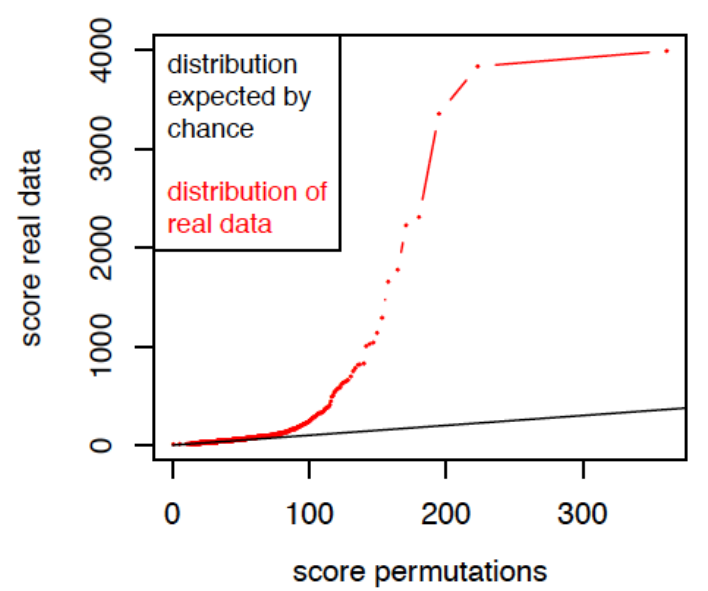

Figure S7. Q-Q plot of expected vs. real FIA eQTL mapping scores. 
A

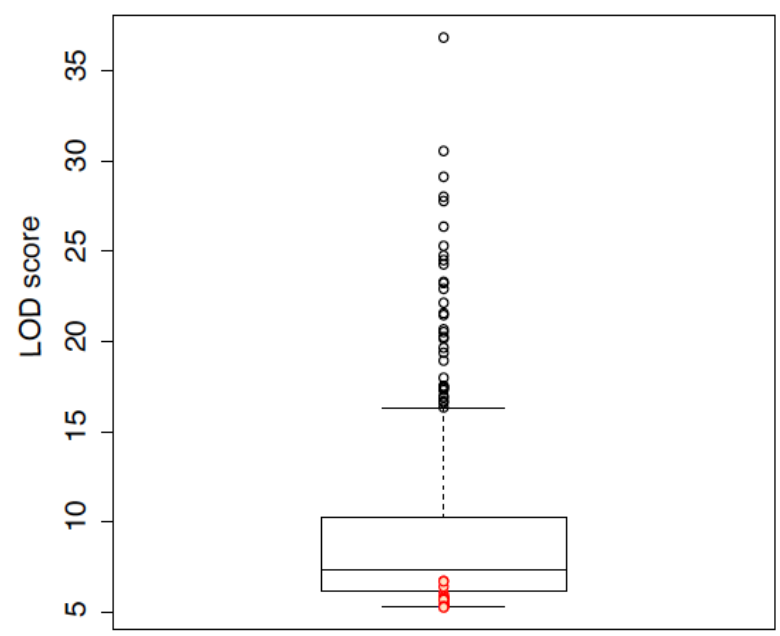

B

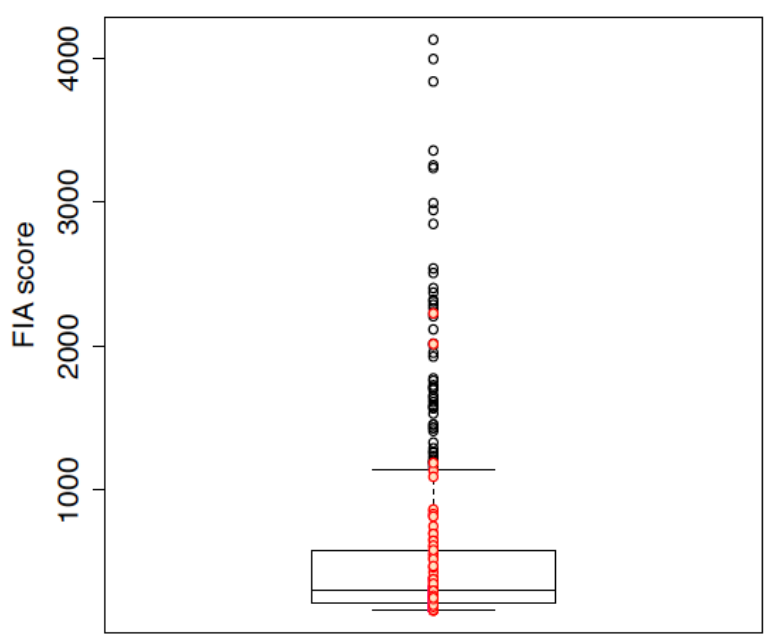

Figure S8. Statistical support for eQTL only found by Haley-Knott Regression (HKR) or Flexible Intercross Analysis (FIA).

A. Boxplot of the LOD scores for all $(n=443)$ eQTL identified by HKR. The LOD scores for eQTL ( $n=32$ ) only detected by HKR (and not by FIA) are shown as red dots. B. Boxplot of the significance scores of all ( $n=689)$ eQTL detected by FIA. The scores for eQTL only detected by FIA ( $n=278$ ) (and not by HKR) are shown as red dots. 


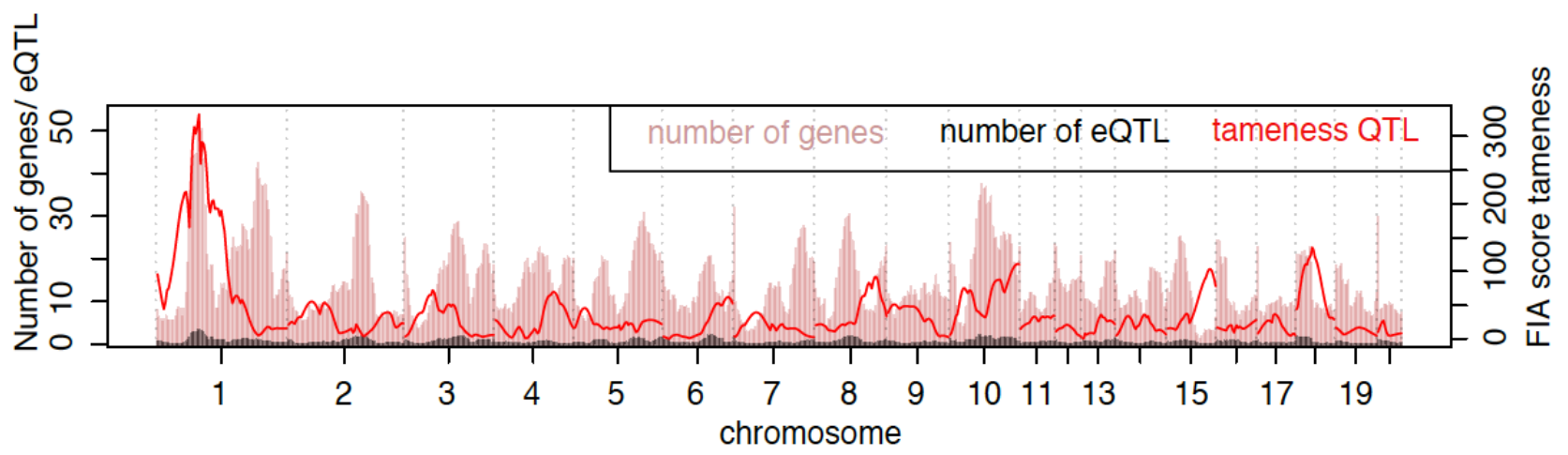

Figure S9. Gene density and statistical support for linkage to tameness along the genome. Light red bars: number of genes. Grey bars: number of local FIA eQTL. Red curve: FIA scores of tameness QTL mapping. 
Detailed Results of Co-expression Network Analysis.

We applied Weighted Gene Co-expression Network Analysis (WGCNA) to investigate whether gene expression traits are clustered in expression networks. We also mapped the "Eigengene" for each cluster (the first principal component of expression level of all genes in a given cluster) to the genome using FIA. Detailed results from the cluster analyses are available in Supplementary Tables S3. The analysis grouped genes into 54 co-expression cluster. Whereas the „Eigengenes“ in five of the clusters were correlated with tameness, none of the QTL influencing these clusters overlapped with tameness QTL. There is therefore currently no evidence that these clusters and tameness are influenced by shared genetic loci. Gene Ontology and transcription factor binding site enrichment analysis of the clusters that correlated with tameness showed enrichment for a large variety of biological processes. For example, the "grey60" cluster was enriched for retrotransposable elements (L1 transposase, FDR $<0.002 \%$ ). Modulation of the transcription of retrotransposable elements occurs in the brain during exposure to stressful environmental conditions, possibly in response to glucocorticoid receptor activation [1]]. The blue cluster was enriched in proteasomal ubiquitin-dependent protein catabolic processes (FDR < 2\%) and enriched for binding sites of transcription factor Elk-1 (FDR < 10e-8). Elk-1 has been shown to be activated subsequently to glucocorticoid receptor activation [2] comforting a possible role of glucorticoids receptors in the formation of the tame-aggressive phenotype.

References to Supplementary Note

1. Hunter, R.G., B.S. McEwen, and D.W. Pfaff, Environmental stress and transposon transcription in the mammalian brain. Mob Genet Elements, 2013. 3(2): p. e24555.

2. Gutierrez-Mecinas, M., et al., Long-lasting behavioral responses to stress involve a direct interaction of glucocorticoid receptors with ERK1/2-MSK1-Elk-1 signaling. Proc Natl Acad Sci U S A, 2011. 108(33): p. 13806-11. 
Supplementary Tables S1-S8

Tables S1-S8 are available for download as Excel sheets at

http://www.genetics.org/lookup/suppl/doi:10.1534/genetics.114.168948/-/DC1 